\title{
Aeroelastic analysis of a floating offshore wind turbine in platform-induced surge motion using a fully coupled CFD-MBD method
}

\author{
Yuanchuan Liu ${ }^{1}$, Qing Xiao ${ }^{1^{*}}$, Atilla Incecik ${ }^{1}$ and Christophe Peyrard ${ }^{2}$ \\ ${ }^{1}$ Department of Naval Architecture, Ocean and Marine Engineering, University \\ of Strathclyde, Glasgow, UK, G4 OLZ \\ ${ }^{2}$ Saint-Venant Hydraulics Laboratory (Électricité de France, ENPC, Cerema), \\ Université Paris-Est, 6 quai Watier, 78400 Chatou, France \\ *Corresponding Author: ging.xiao@strath.ac.uk
}

\begin{abstract}
Modern offshore wind turbines are susceptible to blade deformation due to their increased size and the recent trend of installing these turbines on floating platforms in deep sea. In this paper, an aeroelastic analysis tool for floating offshore wind turbines is presented by coupling a high-fidelity Computational Fluid Dynamics (CFD) solver with a general purpose MultiBody Dynamics code, which is capable of modelling flexible bodies based on the nonlinear beam theory. With the tool developed, we demonstrated its applications to the NREL 5MW offshore wind turbine with aeroelastic blades. The impacts of blade flexibility and platform-induced surge motion on wind turbine aerodynamics and structural responses are studied and illustrated by the CFD results of the flow field, force, and wake structure. Results are compared with data obtained from the engineering tool FAST v8.
\end{abstract}

Keywords: Aeroelastic analysis; Floating offshore wind turbine; Platform-induced surge motion; Computational Fluid Dynamics; MultiBody Dynamics

\section{Introduction}

Wind energy is one of the fastest growing renewable energy sources, with the potential to alleviate environmental problems caused by climate change. The abundance of wind 
resource in offshore areas makes them a popular choice for turbine installation. WindEurope (2017) reports that over 3,500 offshore wind turbines have been installed and connected to the grid by the end of 2016, bringing the cumulative capacity in Europe to over 12.6 GW. Offshore wind turbines are larger in size compared to their onshore counterparts to achieve better power capacity and reduce economic costs. For example, the rotor diameter of an offshore wind turbine with a rated power capacity of 5 MW is over 120 meters (Jonkman et al., 2009). When exposed to unsteady wind, a turbine is subjected to substantial aerodynamic loading, leading to significant blade deformation which in turn influences power generation. On the other hand, repetitive blade deformation due to unsteady cyclic wind loading gives rise to long-term problems such as structural fatigue. The recent trend to install floating offshore wind turbines (FOWT) in deep-water sites makes such structure damage problems more pronounced. For example, the world's first floating wind farm Hywind Scotland consists of five 6MW FOWTs with 75-m long blades, making them more susceptible to structural deformation than fixed-bottom ones. In addition, as an FOWT is installed on a floating platform, six Degrees-of-Freedom (DoF) platform motions directly exert impacts on turbine aerodynamic loading and consequently blade deformation in a periodic manner. As a result, it is necessary to consider both turbine aerodynamics and structural dynamics during the design process and develop an aeroelastic tool to analyse the fluidstructure interaction (FSI) for offshore wind turbines.

A number of tools capable of performing wind turbine aeroelastic analysis have been developed with various orders of accuracy. Based on the methods adopted for aerodynamics and structural dynamics, these tools can be categorised into several groups. Many engineering tools adopt the Blade Element Momentum (BEM) theory for turbine aerodynamics and the modal approach for structural dynamics. Examples are the well-known FAST v7 (Fatigue, Aerodynamics, Structures, and Turbulence) from National Renewable Energy Laboratory (NREL) and FLEX5 developed at Technical University of Denmark (DTU). These codes are highly efficient and suitable for the initial design stage when a large amount of cases need be simulated. The BEM method combines the blade element theory and one-dimensional momentum theory, and solves the induction factor at each element iteratively. It can provide satisfactory results when reasonable sectional airfoil aerodynamic data of lift and drag coefficients is provided (Hansen et al., 2006). The modal approach describes blade deformation as a linear 
combination of several pre-computed mode shapes (Wang et al., 2016). Although it is computationally efficient, the accuracy of the modal approach is limited by the number of DoFs considered. For example, FAST only uses three mode shapes, i.e. $1^{\text {st }}$ and/or $2^{\text {nd }}$ order flapwise and edgewise deflections, which is insufficient for complex situations involving torsional twist. In addition, the linear assumption determines it is not applicable to cases with large blade deflections. To overcome such limitations, other engineering tools like HAWC2 (Larsen and Hansen, 2007) developed by DTU consider blade elasticity using a more sophisticated beam theory. FAST was also recently updated to include a new structural module BeamDyn based on the beam theory (Wang et al., 2017). The beam theory models a three-dimensional turbine blade as a onedimensional beam with varying cross-sectional structural properties. Compared to the modal approach, it can handle more DoFs and take geometric nonlinearities into consideration, thus leading to a better representation of blade deformation. As a result, some recent studies (Jeong et al., 2014; Manolas et al., 2015; Rafiee et al., 2016; Ferede et al., 2017) focused on the coupling of BEM codes with structural solvers based on the beam theory.

Despite the wide adoption of BEM for wind turbine aerodynamics, high-fidelity Computational Fluid Dynamics methods have been increasingly used in recent years due to the rapid advances in computer technology. Compared to BEM, CFD has several advantages. Firstly, CFD does not require accurate input of sectional airfoil lift and drag coefficients as functions of angle of attack (AOA) and/or Reynolds number (Hansen et al., 2006). Instead, it directly models geometrically resolved turbines. Secondly, BEM requires empirical correction models to consider complex three-dimensional flow effects, such as tip-loss and hub-loss corrections for vortices shedding from blade tip and rotor hub, Glauert correction for large induction factors, skewed wake correction for tilt and yaw conditions and dynamic inflow modelling for rapidly changing AOA (Moriarty and Hansen, 2005; Wang et al., 2016). CFD simulations, on the other hand, can inherently take these effects into consideration. Besides, BEM assumes that the forces acting on blade elements are two-dimensional and fluid flow in the spanwise direction is ignored. However, for heavily loaded rotors, spanwise flow along turbine blades cannot be omitted, and can be well predicted by CFD ( $\mathrm{Li}$ et al., 2012). Furthermore, CFD is able to analyse the interaction between a wind turbine and its wake in a floating scenario. 
Regarding structural modelling approaches coupled with CFD, advanced threedimensional finite element methods (FEM) are capable of predicting stress distribution along wind turbines and have been adopted in recent studies (Bazilevs et al., 2011; Hsu and Bazilevs, 2012; Lee et al., 2017). However, as modern turbine blades are constructed with composite materials consisting of various layers, it is often difficult and tedious to create FEM models (Wang et al., 2016). Besides, these methods are relatively more computationally demanding compared to approaches based on the beam theory. On the other hand, for slender structures like turbine blades, the beam theory can usually produce accurate results in structural deformation and internal loading ( $\mathrm{Li}$ et al., 2015). Therefore, the beam theory is still widely used for structural dynamics of flexible blades.

Some researchers have applied coupled CFD and beam codes to investigate wind turbine FSI. As a wind turbine consists of both rigid components, like hub and nacelle, and flexible parts, such as blades and tower, one common approach is to construct a MultiBody Dynamics (MBD) system where a number of bodies, either rigid or flexible, are interconnected via kinematic constraints, while modelling flexible bodies as beams (Pierangelo and Jayanarayanan, 2011; Li et al., 2015; Heinz et al., 2016). Currently, these studies are limited to fixed-bottom wind turbines without considering platform motions in FOWT applications. On the other hand, impacts of platform motions induced by waves and current on wind turbine aerodynamics have been investigated extensively using CFD methods either by imposing prescribed periodic motions to wind turbines (Wu et al., 2015; Leble and Barakos, 2016b; Tran and Kim, 2016a; Wu and Nguyen, 2017) or via performing fully coupled aero-hydrodynamic analysis (Leble and Barakos, 2016a; Tran and Kim, 2016b; Liu et al., 2017b). These CFD studies demonstrated that platform motions greatly affected wind turbine aerodynamics as well as its wake. However, they only focused on wind turbines with rigid blades. To the best of the authors' knowledge, impacts of platform motions on flexible wind turbines have been rarely analysed using a CFD method.

In this paper, a fully coupled numerical tool developed for aeroelastic analysis of offshore wind turbines will be presented. Wind turbine aerodynamics is analysed via a high-fidelity CFD solver based on an open source CFD framework OpenFOAM (https://openfoam.org). Structural dynamics of turbine blades is solved using an open 
source MBD code MBDyn (https://www.mbdyn.org), which is able to model flexible bodies using the geometrically exact beam formulation. These two individual solvers are interfaced using an in-house library developed in OpenFOAM, and a mesh motion library is implemented to deal with the complex CFD grid motion in aeroelastic simulations. The developed tool is applied to the NREL 5MW offshore wind turbine with elastic blades. Effects of blade flexibility on turbine aerodynamics and structural responses are firstly studied with a fixed-bottom wind turbine. Impacts of platforminduced surge motion for an FOWT are subsequently investigated via superimposing a prescribed motion to the turbine. Predicted results are analysed using flow field information from CFD modelling and compared with data from the engineering tool FAST v8.

\section{Numerical Methods}

\subsection{Flow Solver}

The incompressible and turbulent flow of Newtonian fluids with a moving mesh is governed by Eqs. (1)-(2) and solved with the transient CFD solver pimpleDyMFoam in OpenFOAM.

$$
\begin{gathered}
\nabla \cdot \mathbf{U}=0 \\
\frac{\partial \mathbf{U}}{\partial t}+\nabla \cdot\left[\left(\mathbf{U}-\mathbf{U}_{g}\right) \mathbf{U}\right]=-\frac{\nabla p}{\rho}+\nabla \cdot\left[v_{e f f} \nabla \mathbf{U}+(\nabla \mathbf{U})^{T}\right]
\end{gathered}
$$

where $\mathbf{U}$ and $\mathbf{U}_{g}$ represent velocity of flow field and grid nodes, respectively; $p$ is pressure of flow field; $\rho$ is fluid density; $v_{e f f}=v+v_{t}$ denotes effective kinematic viscosity of fluid, in which $v$ and $v_{t}$ are kinematic and eddy viscosity separately.

The two-equation $k-\omega$ shear stress transport (SST) turbulence model (Menter, 2009) is adopted as the closure for the Reynolds-averaged Navier-Stokes (RANS) equations in this study. The $k-\omega$ SST turbulence model combines standard $k-\omega$ and $k-\varepsilon$ models by adopting the standard $k-\omega$ model near the boundary layer and switching to the standard $k-\varepsilon$ model in the far-field. It is suitable for fluid flow with adverse pressure gradients and flow separation and has been widely applied to wind turbine simulations (Leble and Barakos, 2016b; Tran and Kim, 2016a; Wu and Nguyen, 2017). 
The PIMPLE (merged PISO-SIMPLE) algorithm is applied to deal with velocitypressure coupling in a segregated way. A second-order Crank-Nicolson scheme is used for temporal discretisation. A second-order upwind scheme is adopted for convective terms. Gradient terms are handled via a second-order cell-limited Gauss linear scheme.

\subsection{Structural Solver}

Dynamic structural response of a wind turbine is computed using MBDyn. This code adopts a Lagrange multiplier or redundant coordinate set formulation for a multibody system consisting of both rigid and flexible bodies connected by kinematic constraints (Ghiringhelli et al., 2000). For each body of the constrained system, Newton-Euler equations of motion are established in the differential-algebraic form as a set of firstorder equations together with constraint equations as follows:

$$
\begin{array}{r}
\mathbf{M}^{\cdot} \\
\mathrm{l}^{\circ} \quad \mathrm{f}(\mathbf{x}, \\
\phi(\mathbf{x}, t)=0
\end{array}
$$

where $\mathbf{M}$ is inertia matrix; $\mathbf{x}$ represents generalised coordinates including both translational and rotational parameters in the global reference frame; the dot operator above a variable denotes its derivative to time; $\mathbf{p}$ is momentum vector; $\phi$ is a set of kinematic constraints applied on the body and $\phi_{\mathrm{x}}^{\mathrm{T}}$ is the Jacobian of $\phi$ with respect to $\mathbf{x}$; $\mathbf{f}$ is external force vector.

MBDyn models flexible bodies as a series of three-node beam elements based on the geometrically exact beam formulation (Ghiringhelli et al., 2000). As indicated in Figure 1 , one beam element is divided into three portions by two evaluation points (squares). The three portions are associated with three reference points (circles), which represent the elastic axis of the beam. These reference points do not necessarily need be on a straight line and can be offset from the geometrical nodes (triangles) where equilibrium equations are established considering both external and internal forces. External forces are integrated over every beam element portion related to a reference point and later translated to its corresponding geometrical node. Meanwhile, internal forces are evaluated at cross sections of evaluation points and are related to geometrical strains 
and curvatures via constitutive laws. The general linear viscoelastic constitutive law provided in MBDyn is adopted throughout this study and can be described as follows:

$$
\mathbf{F}=\mathbf{K} \boldsymbol{\varepsilon}+\mathbf{C}
$$

where $\mathbf{F}$ represents generalised internal force vector; $\boldsymbol{\varepsilon}$ and $:$ denote generalised strain and strain rate vectors; $\mathbf{K}$ and $\mathbf{C}$ are linear stiffness and viscosity matrices, which need be provided by users for every cross section. A system of equilibrium equations, i.e. Eqs. (3)-(6), is then established and solved using an implicit multistep integration scheme.

\subsection{Mesh Motion Solver}

One of the challenges for fully coupled FOWT simulations with flexible blades is how to handle CFD mesh motion to represent complex structural responses involved. As shown in Figure 2, in addition to global wind turbine rotation and platform-induced 6DoF motions, flexible blades are subjected to local deflections in both out-of-plane (flapwise) and in-plane (edgewise) directions relative to the rotor plane as well as twist deformation along the blade pitch axis. Commonly used sliding mesh and overset grid techniques can only model global motions, while mesh deformation solvers are more suitable for local blade deformation. It is therefore necessary to develop a mesh motion solver able to update CFD mesh both globally and locally. In the present study, a customised mesh motion library is developed in OpenFOAM by incorporating the builtin solid body motion library into the dynamic mesh motion library. The solid body motion library can handle global turbine rotation using Arbitrary Mesh Interface (AMI), i.e. an OpenFOAM implementation of the sliding mesh technique, while the dynamic mesh motion library deals with local blade deformation.

The original dynamic mesh motion solver calculates the displacement of internal mesh cell centres $\mathbf{x}_{g}$ by solving the following Laplace equation (Jasak and Tukovic, 2006):

$$
\nabla \cdot\left(\gamma \nabla \mathbf{x}_{g}\right)=0
$$


where $\gamma=1 / r^{2}$ denotes the diffusion coefficient and $r$ is the distance from cell centres to structure boundaries.

In order to maintain mesh quality, global rigid body motions are firstly subtracted from the actual point displacement of the turbine surface mesh to obtain a relative value, which is then utilised by the mesh motion solver as the boundary condition of Eq. (7). Meanwhile, the AMI surfaces divide the computational domain into two separate cell zones, i.e. the rotor zone encompassing turbine blades and the stator zone, as shown in Figure 3. When the mesh motion equation is assembled, only cells inside the rotor zone are considered while those in the stator zone are kept static by directly setting their displacement to zero. Once the displacement of internal mesh cell centres is obtained, interpolation is performed to obtain the displacement of internal mesh points, which is then added to the initial position of all points to determine their updated position due to local blade deformation. Lastly, these points in the rotor zone are rotated collectively to consider global turbine rotation. If platform-induced 6-DoF motions are also present, they need be applied to both rotor and stator zones. Figure 4 shows a sectional view of the computational mesh before and after blade deformation where grid quality near the blade is mostly preserved. It is worth pointing out that to speed up flexible turbine simulations and minimise CFD mesh distortion due to local blade deformation, aeroelastic turbine blades are pre-bent to an approximate deformed shape as displayed in Figure 2.

\subsection{Coupling Strategy}

As mentioned earlier, a flexible blade is modelled as a series of three-node beam elements in MBDyn, while in CFD it is discretised into a surface grid comprising a large number of surface points, as shown in Figure 5. The gap between the level of complexity in describing the same geometry in two models leads to a pair of unmatched/non-conformal interfaces. Therefore, a numerical scheme to map data between CFD and MBD models is developed in this tool to address this issue.

Figure 5 illustrates the mapping between the two models. In the CFD model, the surface grid of the structure is decomposed into several small patches, each of which corresponds to a beam node in the MBD model. A centre is defined for every patch in 
the CFD grid and has the same kinematics as the corresponding beam node in the MBD model via motion exchange. On the other hand, the external fluid force and moment required by MBDyn are firstly integrated over every patch of the CFD surface grid with respect to its patch centre and then transferred to MBDyn via force exchange.

In order to maintain the smoothness of the surface grid in the CFD model, a linear interpolation scheme (Pierangelo and Jayanarayanan, 2011; Li et al., 2015) is implemented in the present tool to calculate the position of each point on the CFD surface grid using the kinematics from the centre of the patch it is on and the centre of its adjacent patch. For example, point A (cross) in Figure 5 uses kinematics of patch centres $i$ and $i+1$ while point B (square) needs information from patches $i$ and $i-1$. The following formula describes the interpolation scheme for the position of point $\mathrm{A}$ :

$$
\mathbf{X}=\xi\left(\mathbf{X}_{i}+\mathbf{R}_{i} \mathbf{d}_{i}\right)+(1-\xi)\left(\mathbf{X}_{i+1}+\mathbf{R}_{i+1} \mathbf{d}_{i+1}\right)
$$

where $\mathbf{X}$ represents position of point or patch centre; $\mathbf{R}$ denotes transformation matrix of patch centre due to rotation; $\mathbf{d}$ is distance vector pointing from patch centre to point; $\xi \in[0,1]$ stands for normalised point location between surrounding patch centres.

When a fully coupled simulation is performed, the CFD solver runs in parallel to take advantage of High Performance Computing (HPC) while MBDyn runs simultaneously in serial as an individual computer process. Data exchange between two codes is achieved with the help of TCP/IP (Transmission Control Protocol/Internet Protocol) communication protocol, using a client/server model. An interface library is implemented in OpenFOAM by adopting motion and force exchange functions provided in MBDyn, serving as the bridge connecting flow and structural solvers.

Figure 6 shows the coupling strategy used in the present tool. At the start of a simulation, MBDyn creates a TCP/IP socket and acts as a server, while pimpleDyMFoam connects to the socket as a client, establishing a two-way communication. In general, fluid force and moment for every patch are integrated in pimpleDyMFoam while MBDyn waits for the data to be transferred. After receiving the force data, MBDyn calculates the structural response of the system, i.e. the kinematics of the geometric nodes, and then transfers the structural motion data back to the CFD solver. Compared to the time the 
CFD code requires to solve fluid flow, MBDyn is highly efficient and the idle time of pimpleDyMFoam while waiting for the motion data from MBDyn is negligible. The CFD mesh is then updated in the mesh motion solver based on the mapped point position from the motion data of each patch centre, followed by an update of flow field. As both codes solve equations using iterative approaches, the communication between OpenFOAM and MBDyn is completed at each iteration so that a strong coupling is achieved with robust and quick convergence. Within each time step, data exchange is performed between OpenFOAM and MBDyn for typically 5 iterations until flow field eventually converges. MBDyn then continues its iteration with the external loading received from the last data transfer until converged structural responses are obtained.

\section{Model Description}

The fully coupled FSI analysis tool was validated in our previous work (Liu et al., 2017a) with a $2 \mathrm{D}$ benchmark case of a flexible cantilever beam attached to a rigid square cylinder in free stream. In the present study, the NREL 5MW offshore wind turbine is analysed as shown in Figure 2. This model is selected because it is specifically designed for offshore applications and has been widely used as benchmark by researchers in the offshore wind energy field. Detailed information about the wind turbine geometry and structural properties can be found in the NREL report (Jonkman et al., 2009). It should be noted that some modifications are made to simplify our CFD modelling. Firstly, only turbine blades are modelled while tower, nacelle and hub are ignored. Besides, the shaft tilt angle is set to zero while the pre-cone angle is kept as $2.5^{\circ}$. Due to the lack of experiment associated with aeroelastic turbine blades, the engineering tool FAST v8 is adopted in the present study to compare results obtained with a high-fidelity code and a reduced order model. Same settings are applied to simulations performed with the present tool and FAST v8.

\subsection{CFD Model}

Figure 7 illustrates the computational domain and boundary conditions in our CFD simulations. Constant wind speed is imposed for flow velocity at the Inlet boundary while zero pressure is applied at the Outlet boundary. The Neumann boundary condition is assumed for both velocity and pressure at all four Farfield boundaries, and a no-slip moving wall boundary condition is imposed on the rotating blades. 
A mesh-sensitivity test is carried out by setting up four cases with different grid density, as listed in Table 1, under the working condition with a wind speed of $8 \mathrm{~m} / \mathrm{s}$, rotor speed of 9 RPM and blade pitch angle of $0^{\circ}$. The built-in utility snappyHexMesh in OpenFOAM is adopted to generate a hexahedra-dominant unstructured grid in three successive steps: splitting cells of a hexahedra background mesh in user-specified regions, snapping cells to surface geometry and adding mesh layers from surface boundaries. Please refer to its user manual (OpenFOAM, 2013) for a detailed explanation. The four grids are generated by adopting background hexahedra meshes with different cell sizes while keeping other parameters such as cell splitting levels and the height of first cell unchanged. Figure 8(a) shows the mesh refinement applied for Grid \#3 near blade root and tip regions to better capture root and tip vortices. Figure 8(b) demonstrates the surface mesh on a turbine blade and more cells are distributed towards the leading and trailing edges to better describe the geometry and predict the rapid variance of fluid flow. Figure 8(c) illustrates the refined mesh near a turbine blade section, where ten layers of boundary layer cells are extruded from the turbine surface mesh with a growth ratio of 1.3 . The height of the first cell away from the turbine surface is set to $0.0025 \mathrm{~m}$ to ensure that $\mathrm{y}+$ is within the range of $[30,300]$ and wall functions are adopted for near wall treatment in the $k-\omega$ SST turbulence model. To speed up computation, the built-in steady-state solver simpleFoam with the Multiple Reference Frame (MRF) functionality in OpenFOAM is utilised where velocity is firstly solved in a relative reference frame rotating with the turbine and then transformed to a stationary inertial reference frame without rotating the computational mesh. Table 1 summarises converged turbine thrust and power for all cases. It is shown that the difference of both thrust and power between the finest Grid \#4 and Grid \#3 is less than $2 \%$, and Grid \#3 is thus selected for subsequent simulations.

Using the optimal grid obtained above, simulations for a fixed-bottom wind turbine under a series of working conditions listed in Table 2 are conducted as benchmark tests. Although it is computationally efficient, the steady-state MRF approach cannot take into consideration the unsteadiness in fluid flow associated with turbulence and wake dynamics (Wu and Nguyen, 2017). In order to achieve better accuracy, the unsteady flow solver pimpleDyMFoam is adopted together with the sliding mesh or AMI technique. Nonetheless, steady-state results are employed to serve as the initial conditions for unsteady simulations to skip the start-up stage and thus save 
computational time. The time step size $\Delta t$ is set to satisfy $\omega \Delta t=0.4 \mathrm{deg}$, i.e. 0.4 degrees per time step. Figure 9 compares the time-averaged wind turbine thrust and power over the last rotation cycle under various working conditions obtained by present and previous CFD simulations (Sørensen and Johansen, 2007; Chow and van Dam, 2012; Wu and Nguyen, 2017) as well as FAST v8. It is shown that similar trends are predicted by different approaches and present results agree reasonably well with published data. At the rated operating condition with a wind speed of $11.4 \mathrm{~m} / \mathrm{s}$, the present tool predict a maximum thrust of $746 \mathrm{kN}$ which falls within the range from 690 $\mathrm{kN}$ to $760 \mathrm{kN}$ calculated from other simulations. The discrepancies between CFD results could be associated with differences in the numerical methods adopted by each code, such as discretisation schemes and solving procedures.

\subsection{Structural Model}

The structural model used in MBDyn for one turbine blade is illustrated in Figure 10. All the structural properties used in this study, e.g. inertia and sectional stiffness, are extracted from the NREL report (Jonkman et al., 2009). Twenty-five three-node beam elements, i.e. 49 geometrical nodes in total, are used to represent one blade. More nodes are positioned towards blade root and tip to better describe the rapid variation of geometrical shape and structural properties in these regions. The turbine hub is treated as a rigid body with only one node. A separate static or ground node is also used as a reference node for other nodes. For a three-bladed turbine, the total number of geometrical nodes reaches 149 .

The hub node is forced to rotate relative to the static ground node along the rotation axis at a specified turbine rotation speed. The node at the blade root and the hub node are clamped together with no relative translational and rotational motions, so the blade root node moves with the rotating hub node. Subsequently, all other blade nodes also rotate due to the restraint imposed by the beam elements they are associated with.

The structural model is compared with the nonlinear structural solver BeamDyn (Wang et al., 2017) developed and validated in FAST v8. A test case is set up, where aerodynamic loading is not considered and only a concentrated loading of $100 \mathrm{kN}$ is applied at the blade tip node in the flapwise direction. Figure 11 shows blade deflection in both flapwise and edgewise directions. MBDyn predicted a maximum flapwise 
deflection of $7.47 \mathrm{~m}$ and an edgewise deflection of $-0.41 \mathrm{~m}$ at its tip, while for BeamDyn they are $7.37 \mathrm{~m}$ and $-0.415 \mathrm{~m}$, respectively. Good agreement is achieved between results obtained with MBDyn and BeamDyn, indicating that the current structural model can be used for subsequent aeroelastic simulations.

\subsection{Simulation Cases}

Table 3 summarises the simulation cases investigated in the present study. In Case \#1, the turbine base is fixed to represent a fixed-bottom wind turbine installed nearshore in shallow water. A sinusoidal translational motion parallel to the wind direction is imposed upon the turbine base for Case \#2 to analyse the influence of platform surge motion associated with an FOWT scenario. Although a platform has six DoF motions, focusing on pure surge motion in this study makes it easy to analyse flow field and force, thus providing more insights as compared to a more complicated six DoF case. The oscillating surge motion has an amplitude of $2 \mathrm{~m}$ and a time period of $12 \mathrm{~s}$, which is extracted from the work of Wu et al. (2015) for subsequent qualitative comparisons. Figure 12 shows the surge displacement and velocity over one cycle. For both cases, simulations with rigid and flexible turbine blades are carried out to investigate effects of blade flexibility.

The rated wind turbine working condition, i.e. wind speed of $11.4 \mathrm{~m} / \mathrm{s}$ and rotor speed of 12.1 RPM, is applied in both cases. Under such flow condition, turbine aerodynamic thrust reaches its maximum as indicated in Figure 9, and the difference between various cases can be best illustrated.

\section{Results and Discussion}

\subsection{Blade Flexibility}

In this section, the influence of blade flexibility is investigated by comparing one rigid turbine case and another with flexible blades under Case \#1 condition. Both simulations are run for a few turbine rotations using the transient solver pimpleDyMFoam until the aerodynamic loading acting upon the turbine varies within $0.1 \%$ over one rotation cycle. A quasi-steady state is then assumed to have been achieved and time-averaged data over the last full cycle is extracted for analysis. 
Table 4 summarises predicted wind turbine thrust and power using the present coupled CFD-MBD tool. It is shown that the thrust associated with flexible blades decreases by $1.7 \%$ compared to the rigid case, and power is $3.1 \%$ smaller. Results calculated using FAST v8 are also listed in Table 4 for comparison. It should be noted that the control module in FAST v8 is not activated throughout this study. The aerodynamic module AeroDyn v15 based on the BEM theory is employed for turbine aerodynamics, and the nonlinear structural solver BeamDyn introduced in FAST v8 is adopted for structural dynamics. FAST v8 predicts a decrease of 3.3\% in thrust for flexible blades compared to the rigid case, which is in good agreement with results from the present tool. The power obtained from FAST v8 in the flexible turbine case is $3.9 \%$ smaller than in the rigid case, which is also consistent with present results. Similar trends were also observed in the work of Yu and Kwon (2014) where deformation of blades resulted in reduced aerodynamic thrust and power for a fixed-bottom turbine.

Figure 13(a) illustrates the spanwise distribution of blade deflections in flapwise and edgewise directions. Deflections at blade tip are summarised in Table 5. The present CFD-MBD approach predicts a blade tip deflection of 5.6m in flapwise direction, which is much larger than the edgewise deflection of $0.6 \mathrm{~m}$. This can be attributed to larger structural bending stiffness in edgewise than flapwise direction according to data provided by Jonkman et al. (2009). The edgewise deflection is defined along airfoil chord line, positive from the leading edge to its trailing edge. The negative edgewise deflection in Table 5 indicates that the blade deforms from the trailing edge towards the leading edge, which is induced by the aerodynamic torque in the same direction as turbine rotation. FAST v8 also predicts similar results to the present tool.

The bending moment distribution in spanwise direction for the above case is displayed in Figure 13(b). It represents the internal structural moment at each section along the blade obtained from the structural stress-strain constitutive law. The bending moment is much larger in flapwise than in edgewise direction throughout the blade. At blade root, the flapwise bending moment increases to $10 \mathrm{MN}^{*} \mathrm{~m}$, nearly 10 times the edgewise bending moment, which further explains the significantly larger deflection in flapwise than edgewise deflection. 
To better assess the influence of blade flexibility on turbine aerodynamics, in the following section, we will utilise our CFD results to explore the potential reasons behind the difference between rigid and flexible cases. Figure 14 displays the spanwise distribution of blade thrust and power. It is shown that there is a noticeable discrepancy around peak region between $0.75 \mathrm{R}$ and $0.95 \mathrm{R}$, near blade tip. A flexible blade has smaller thrust and power than a rigid one, which explains the decrease of overall turbine aerodynamic performance.

To further examine the underlying causes for the decrease, the pressure coefficient $\mathrm{Cp}$ distribution at the blade spanwise section of 0.9R is plotted in Figure 15(a). Although the two curves closely resemble each other, a pressure drop (circle) near the leading edge on the suction side of airfoil is present. This could be associated with blade deformation, mainly the twist deflection shown in Figure 15(b). The blade twist deflection, which results from the torsional loading acting on the blade, should not be mixed up with the aerodynamic twist, which is from the blade design specification, although they both affect the apparent or effective AOA of an airfoil by altering its chord-line orientation as illustrated in Figure 15(c). It is worth noting that the estimation of apparent AOA does not include the induced wind speed in axial and tangential directions in the incoming wind speed $U_{\text {Wind }}$ due to the inherent challenges to quantitatively define them as discussed previously by Li et al. (2015). Nonetheless, using this approach can still help understand effects of blade flexibility in a qualitative way. Figure 15(b) shows twist deflection is mostly negative for the deformed blade, indicating that airfoil sections experience nose-down rotations ( $\mathrm{Yu}$ and Kwon, 2014), which reduces the apparent AOA. Within the spanwise region from $0.75 \mathrm{R}$ to $0.95 \mathrm{R}$, the geometrical airfoil shape of blade sections is NACA64 and the apparent AOA is around 8 degrees. As the stall angle for this airfoil is about 13.5 degrees according to its aerodynamics data (Jonkman et al., 2009), a decrease in apparent AOA induced by twist deflection leads to the reduction of lift force and consequently thrust and power.

\subsection{Platform Surge Motion}

In this section, the sinusoidal translational motion in Case \#2 is applied to the wind turbine to investigate the impacts of platform surge motion. Both rigid and flexible blades were simulated for 60 s, i.e. five platform motion cycles, until the variation of turbine aerodynamic loading reached a periodic state. Simulations were performed on 
a cluster with 30 compute nodes, each of which contains two $2.7 \mathrm{GHz}, 12$-core Intel E5-2697 v2 CPUs. The overall CPU time for the rigid case was approximately 42 hours while it took the flexible simulation almost 96 hours to finish. The increase in computational time could be related to the additional mesh morphing equations introduced to consider blade deflection and coupling between the two codes.

The time history data for aerodynamic thrust and power is plotted in Figure 16, along with results from FAST v8 and another CFD study for a rigid turbine by Wu et al. (2015) for comparison. It is shown that both thrust and power vary significantly with respect to time once the platform motion is superimposed. For example, the power predicted by the present tool for the flexible case ranges from $3.85 \mathrm{MW}$ to $6.21 \mathrm{MW}$, i.e. a variation of more than $20 \%$ compared to the fixed-bottom turbine case.

To further compare results from different analysis tools, the amplitudes of the variation of both thrust and power are shown in Figure 17, which is defined as (Max - Min)/2, where Max and Min are the maximum and minimum of either thrust or power over one platform motion cycle, respectively. Comparing the variation amplitude of thrust for the three rigid cases reveals that FAST v8 predicts 10-20\% larger thrust variation than the other two CFD tools. The discrepancy among the results from different tools is also present for power variation. A likely reason for such difference is that CFD tools inherently take into account platform motion effects on the turbine wake via solving Navier-Stokes equations, whereas the BEM theory adopted by FAST v8 utilises an empirical dynamic wake model.

The variation of wind turbine aerodynamic loading under platform motion can again be associated with the change in the apparent AOA in a similar way as described in Section 4.1. Figure 15(c) shows that the apparent AOA is directly related to the wind speed $\mathrm{U}_{\text {Wind }}$ experienced by a blade airfoil section. Increasing $U_{\text {Wind }}$ while maintaining turbine rotation speed $U_{R o t}$, leads to an increase in the relative wind speed $U_{\text {RelWind }}$ experienced by the airfoil section as well as in its apparent AOA. When a platform surge motion is imposed to the turbine, a platform surge velocity $U_{\text {Surge, }}$ shown in Figure 12, must be subtracted from $U_{\text {Wind, }}$, resulting in an apparent wind speed $U_{\text {WindApp }}=U_{\text {Wind }}-U_{\text {Surge. }}$ At the beginning of a platform motion cycle, the turbine is at an equilibrium position with a maximum surge velocity $U_{\text {Surge }}$ in the downwind direction. As a result, $U_{\text {WindApp }}$ is at 
its minimum, effectively leading to a minimum $U_{\text {RelWind }}$ and apparent AOA. Consequently, turbine thrust and power reach their minima. Similarly, maximum turbine aerodynamic loading occurs at half of the platform motion cycle due to the increase in $\mathrm{U}_{\text {RelWind }}$ and apparent $\mathrm{AOA}$ associated with $\mathrm{U}_{\text {Surge. }}$

The difference in thrust and power variation amplitudes between rigid and flexible turbine simulations is relatively small. For example, the variation of thrust predicted by the present tool for the flexible case is about $5 \%$ larger than that for the rigid one, while the difference in power variation is less than $1 \%$. For comparison, FAST v8 predicts a decrease of $0.5 \%$ and $4 \%$ for thrust and power variation, respectively. However, there is a noticeable phase difference in the time history of the flexible case in comparison with the rigid one. This is exemplified by highlighting the maxima and minima of CFD results for rigid and flexible cases with circular $(\bullet)$ and square $(\boldsymbol{\bullet})$ markers, respectively, as shown in Figure 16. It can be observed that the time when either the maximum or minimum thrust occurs for a flexible blade always comes later than a rigid blade, similar to results from FAST v8. Since all other parameters are the same for the rigid and flexible cases, this noticeable time-lag in wind turbine aerodynamic loading is directly related to the deflection of turbine blades.

To better analyse the influence of blade deflection on turbine aerodynamics, the blade element at the section of $0.9 \mathrm{R}$ in the spanwise direction is investigated where maximum thrust and power per unit length are generated in the fixed-bottom condition as shown in Figure 14. Figure 18(a) demonstrates the time history of thrust variation per unit length at $0.9 \mathrm{R}$ for both rigid and flexible cases, as well as the apparent wind speed $\mathrm{U}_{\text {WindApp }}=\mathrm{U}_{\text {Wind }}-\mathrm{U}_{\text {Surge }}-\mathrm{U}_{\text {FlapDef. }}$ Compared to the rigid case, an additional blade

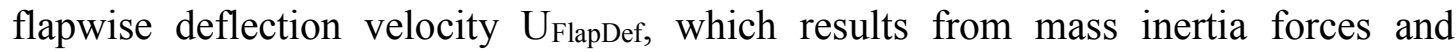
periodically varying aerodynamic loading, has to be considered for the flexible case. As shown in Figure 18(a), the flexible $U_{\text {WindApp }}$ has similar magnitude to its rigid counterpart, indicating that $U_{\text {FlapDef }}$ is much smaller than $U_{\text {Surge. }}$ Nevertheless, thrust for the flexible blade is in phase with the corresponding apparent wind speed $U_{\text {WindApp, }}$ which confirms that the time-lag results from $U_{\text {FlapDef. }}$

In addition to the phase difference between rigid and flexible cases, the gap between the minimum thrust in the rigid case and that in the flexible one is larger than the 
difference for the maximum values, as annotated in Figure 18(a), which can be associated with twist deflection. Figure 18(b) shows the time history of twist deflection at spanwise section of 0.9R as well as the apparent AOA defined in Figure 15(c). The twist deflection is also in phase with the flexible thrust. Near the start of the platform motion cycle, the twist deflection reaches its maximum in the negative direction at 0.46 degrees and gradually decreases to -0.1 degrees after half a cycle. As stated previously in Section 4.1, negative twist deflection reduces the apparent AOA. Therefore, the variation of twist deflection leads to the difference of the apparent AOA between flexible and rigid cases, and accordingly thrust.

Figure 19(a) shows the pressure coefficient Cp distribution at the spanwise section of $0.9 \mathrm{R}$ for the flexible case. Data from two time instants is compared, i.e. when thrust reaches its minimum and maximum. Significant variation of $\mathrm{Cp}$ is observed near the leading edge of the airfoil on both pressure and suction surfaces. Compared to the instant when maximum thrust is achieved, the decrease in $\mathrm{Cp}$ at its minimum spans from the leftmost leading edge to $60 \%$ of the chord length. Figure 19(b) compares the fluid field coloured by $\mathrm{Cp}$ at the spanwise section of $0.9 \mathrm{R}$ for these two instants. The difference in $\mathrm{Cp}$ is clearly visible near the leading edge and above the suction surface, demonstrating the impacts of platform motion on fluid flow around wind turbine.

Figure 20 illustrates the evolution of fluid flow over one platform motion cycle. The vortical structures of the flow field are represented by the contour of second invariant of the rate of strain tensor Q, and coloured by the axial component of fluid flow velocity Ux. As clearly seen from these figures, strong vortices appear near blade tip and root regions. The number and size of these vortex tubes vary considerably at different instants, indicating that the turbine violently interacts with its wake due to the prescribed platform motion, which was also observed by Tran and Kim (2016a) for a wind turbine with rigid blades.

The deflected blade shape at its minimum and maximum flapwise deformation is illustrated in Figure 21(a) and compared to the rigid blade, demonstrating the significant variation in blade flapwise deflection. Figure 21(b) shows the time history of blade tip deflection in flapwise and edgewise directions. The flapwsie and edgewise blade tip deflections oscillate considerably due to the unsteady aerodynamic forces as well as 
inertia loads induced by the prescribed platform motion. The prescribed platform motion also influences the blade bending moment. Figure 21(c) plots the time history of the blade root bending moment in flapwise and edgewise directions. The cyclic characteristic of the internal loading can also be associated with the unsteady aerodynamic forces and the periodic platform motion. In real-world applications, complex working conditions, such as turbulent wind and irregular waves, and multiple DoF platform motion responses could lead to significant variations in wind turbine internal loading and subsequently severe structural problems like fatigue. It is thus necessary to take wind turbine FSI into consideration during the design process.

\section{Conclusions}

This paper presents a fully coupled FSI tool with its application in offshore wind turbine aeroelastic analysis. In particular, wind turbine aerodynamics is solved using a highfidelity CFD solver and structural dynamics of flexible blades is handled by an MBD code, which is able to model flexible bodies based on a nonlinear beam theory. An inhouse interface code is developed to enable the exchange of fluid force and structural response between the two separate solvers. A dynamic mesh motion library is also implemented to tackle the complex CFD grid motion in aeroelastic simulations. The NREL 5MW offshore wind turbine was investigated using the present tool.

With the above-mentioned CFD-MBD tool, we analysed two important problems, i.e. impacts of blade elasticity and influence of platform surge motion. Blade flexibility was investigated by comparing two cases with either rigid or flexible blades while the turbine base is fixed. It was found that deformation of flexible blades reduced wind turbine aerodynamic thrust and power by less than 5\%. Significant flapwise blade tip deflection as large as $8.89 \%$ of rotor radius was predicted. Similar results were also obtained from the engineering tool FAST v8. The decrease in turbine aerodynamic performance for the flexible case was confirmed by inspecting the spanwise thrust and power distribution along a blade and the pressure distribution around an airfoil section. Subsequent analysis revealed that the decrease was associated with the nose-down rotation due to blade twist deformation, which effectively reduced the apparent AOA.

A sinusoidal translational motion was then prescribed to the turbine base to study the effects of platform surge motion on an FOWT with flexible blades. Wind turbine 
aerodynamic thrust and power varied considerably when platform surge motion was imposed. However, FAST v8 overpredicted the variation of turbine aerodynamic thrust and power by $10-20 \%$ compared to CFD simulations, possibly due to the empirical dynamic wake model it used. The variations in turbine thrust and power were attributed to the additional superimposed surge velocity which influenced the apparent wind speed and consequently the apparent AOA. The induced blade deflection velocity in the flapwise direction further changed the apparent wind speed experienced by the flexible blade, leading to the time-lag between its aerodynamic loadings and the prescribed motion. Meanwhile, analysis of the thrust per unit length at blade spanwise section of $0.9 \mathrm{R}$ demonstrated the effects of the varying blade twist deflection due to platform motion. Visualisation of fluid pressure obtained from CFD analysis clearly showed the influence of platform motion on the fluid field around the wind turbine, which cannot be predicted using engineering tools like FAST. Large variations were also observed for blade tip deflections and root bending moments in flapwise and edgewise directions.

Although the present study is limited to pure surge motion of a 5MW FOWT, the aeroelastic tool developed in this work can be further applied to other platform motions, such as pitch and yaw, and larger turbines like the DTU 10MW wind turbine (Bak et al., 2013) in future studies. Effects of platform motion parameters, i.e. amplitude and period, and wind turbine rotational speed can also be investigated. Other potential applications of the present tool include aeroelastic analysis of wake interference effects on the downstream wind turbine in a two-turbine configuration, which is beyond the capability of the BEM theory used by engineering tools. When it is combined with the previously developed aero-hydro-mooring CFD tool for FOWT simulations under wind-wave conditions (Liu et al., 2017b), it becomes possible to perform fully coupled aero-hydro-mooring-elastic analysis for FOWTs under the CFD-MBD framework, which can provide high-fidelity results to calibrate BEM-based engineering tools currently adopted in FOWT projects. Besides, as modern wind turbines are equipped with sophisticated control systems to optimise power output, it is necessary to integrate a control system module with the present tool to carry out more realistic simulations.

\section{Acknowledgements}

Results were obtained using the EPSRC funded ARCHIE-WeSt High Performance Computer (www.archie-west.ac.uk). EPSRC grant No. EP/K000586/1. The first author 
would like to acknowledge Électricité de France (EDF) for providing a scholarship for his $\mathrm{PhD}$ research and access to its Athos HPC facility. The authors are also grateful to the UK Royal Society and China National Science Foundation Council (NSFC) for partial support of this work. Royal Society grant No. IE131059.

\section{References}

Bak C, Zahle F, Bitsche R, Kim T, Yde A, Henriksen LC, Hansen MH, Blasques JPAA, Gaunaa M, Natarajan A (2013). The DTU 10-MW Reference Wind Turbine. Danish Wind Power Research 2013, Fredericia, Denmark.

Bazilevs Y, Hsu MC, Kiendl J, Wüchner R, Bletzinger KU (2011). 3D simulation of wind turbine rotors at full scale. Part II: Fluid-structure interaction modeling with composite blades. International Journal for Numerical Methods in Fluids, 65(1-3), 236-253.

Chow R, van Dam CP (2012). Verification of computational simulations of the NREL $5 \mathrm{MW}$ rotor with a focus on inboard flow separation. Wind Energy, 15(8), 967981.

Ferede E, Abdalla MM, van Bussel GJW (2017). Isogeometric based framework for aeroelastic wind turbine blade analysis. Wind Energy, 20(2), 193-210.

Ghiringhelli GL, Masarati P, Mantegazza P (2000). Multibody Implementation of Finite Volume C Beams. AIAA Journal, 38(1), 131-138.

Hansen MOL, Sørensen JN, Voutsinas S, Sørensen N, Madsen HA (2006). State of the art in wind turbine aerodynamics and aeroelasticity. Progress in Aerospace Sciences, 42(4), 285-330.

Heinz JC, Sørensen NN, Zahle F, Skrzypiński W (2016). Vortex-induced vibrations on a modern wind turbine blade. Wind Energy, 19(11), 2041-2051.

Hsu M-C, Bazilevs Y (2012). Fluid-structure interaction modeling of wind turbines: simulating the full machine. Computational Mechanics, 50(6), 821-833.

Jasak H, Tukovic Z (2006). Automatic mesh motion for the unstructured finite volume method. Transactions of FAMENA, 30(2), 1-20.

Jeong M-S, Cha M-C, Kim S-W, Lee I, Kim T (2014). Effects of torsional degree of freedom, geometric nonlinearity, and gravity on aeroelastic behavior of largescale horizontal axis wind turbine blades under varying wind speed conditions. Journal of Renewable and Sustainable Energy, 6(2), 19.

Jonkman JM, Butterfield S, Musial W, Scott G. Definition of a 5-MW reference wind turbine for offshore system development. 2009: 63.

Larsen TJ, Hansen AM. How 2 HAWC2, the user's manual. 2007.

Leble V, Barakos G (2016a). Demonstration of a coupled floating offshore wind turbine analysis with high-fidelity methods. Journal of Fluids and Structures, 62, 272293.

Leble V, Barakos G (2016b). Forced pitch motion of wind turbines. Journal of Physics: Conference Series, 753(2), 022042. 
Lee K, Huque Z, Kommalapati R, Han S-E (2017). Fluid-structure interaction analysis of NREL phase VI wind turbine: Aerodynamic force evaluation and structural analysis using FSI analysis. Renewable Energy, 113, 512-531.

Li Y, Castro AM, Sinokrot T, Prescott W, Carrica PM (2015). Coupled multi-body dynamics and CFD for wind turbine simulation including explicit wind turbulence. Renewable Energy, 76, 338-361.

Li Y, Paik K-J, Xing T, Carrica PM (2012). Dynamic overset CFD simulations of wind turbine aerodynamics. Renewable Energy, 37(1), 285-298.

Liu Y, Xiao Q, Incecik A (2017a). A coupled CFD/Multibody Dynamics analysis tool for offshore wind turbines with aeroelastic blades. 36th International Conference on Ocean, Offshore and Arctic Engineering, Trondheim, Norway, OMAE2017-61062.

Liu Y, Xiao Q, Incecik A, Peyrard C, Wan D (2017b). Establishing a fully coupled CFD analysis tool for floating offshore wind turbines. Renewable Energy, 112, 280-301.

Manolas DI, Riziotis VA, Voutsinas SG (2015). Assessing the Importance of Geometric Nonlinear Effects in the Prediction of Wind Turbine Blade Loads. Journal of Computational and Nonlinear Dynamics, 10(4), 041008-041008-15.

Menter FR (2009). Review of the shear-stress transport turbulence model experience from an industrial perspective. International Journal of Computational Fluid Dynamics, 23(4), 305-316.

Moriarty PJ, Hansen AC. AeroDyn Theory Manual. 2005.

OpenFOAM. Mesh generation with the snappyHexMesh utility. 2013. Available from: http://www.openfoam.org/docs/user/snappyHexMesh.php\#x26-1510005.4.

Pierangelo M, Jayanarayanan S (2011). Coupled CFD/Multibody Analysis of NREL Unsteady Aerodynamic Experiment Phase VI Rotor. 49th AIAA Aerospace Sciences Meeting including the New Horizons Forum and Aerospace Exposition, Orlando, Florida, USA.

Rafiee R, Moradi M, Khanpour M (2016). The influence of material properties on the aeroelastic behavior of a composite wind turbine blade. Journal of Renewable and Sustainable Energy, 8(6), 063305.

Sørensen NN, Johansen J (2007). UPWIND, aerodynamics and aero-elasticity rotor aerodynamics in atmospheric shear flow. European Wind Energy Conference, Milan, Italy.

Tran TT, Kim D-H (2016a). A CFD study into the influence of unsteady aerodynamic interference on wind turbine surge motion. Renewable Energy, 90, 204-228.

Tran TT, Kim D-H (2016b). Fully coupled aero-hydrodynamic analysis of a semisubmersible FOWT using a dynamic fluid body interaction approach. Renewable Energy, 92, 244-261.

Wang L, Liu X, Kolios A (2016). State of the art in the aeroelasticity of wind turbine blades: Aeroelastic modelling. Renewable and Sustainable Energy Reviews, 64, 195-210. 
Wang Q, Sprague MA, Jonkman J, Johnson N, Jonkman B (2017). BeamDyn: a highfidelity wind turbine blade solver in the FAST modular framework. Wind Energy, 20(8), 1439-1462.

WindEurope. The European offshore wind industry: Key trends and statistics 2016. 2017.

Wu C-HK, Nguyen V-T (2017). Aerodynamic simulations of offshore floating wind turbine in platform-induced pitching motion. Wind Energy, 20(5), 835-858.

Wu J, Ding J-h, He Y-p, Zhao Y-s (2015). Study on Unsteady Aerodynamic Performance of Floating Offshore Wind Turbine by CFD Method. The Twentyfifth International Offshore and Polar Engineering Conference, Kona, Hawaii, USA, p. 554-560.

Yu DO, Kwon OJ (2014). Predicting wind turbine blade loads and aeroelastic response using a coupled CFD-CSD method. Renewable Energy, 70, 184-196. 
Table 1 Mesh-sensitivity test with wind speed of $8 \mathrm{~m} / \mathrm{s}$, rotor speed of $9 \mathrm{RPM}$ and blade pitch angle of $0^{\circ}$ (percentage in parentheses shows difference over data obtained with coarser grid above)

\begin{tabular}{|c|c|c|c|c|}
\hline Grid & $\begin{array}{c}\text { Background Mesh } \\
\text { Cell Size }(\mathrm{m})\end{array}$ & $\begin{array}{c}\text { Cell Number } \\
\text { (in million) }\end{array}$ & Thrust (kN) & Power (MW) \\
\hline 1 & 30 & 2.22 & 341 & 1.367 \\
\hline 2 & 20 & 4.36 & $357(+4.7 \%)$ & $1.612(+17.9 \%)$ \\
\hline 3 & 15 & 7.44 & $360(+0.8 \%)$ & $1.678(+4.1 \%)$ \\
\hline 4 & 12 & 11.6 & $362(+0.6 \%)$ & $1.706(+1.7 \%)$ \\
\hline
\end{tabular}

Table 2 Specifications for working conditions in benchmark tests

\begin{tabular}{|c|c|c|}
\hline Wind Speed U (m/s) & Rotor Speed (RPM) & Blade Pitch (degrees) \\
\hline 8 & 9 & 0 \\
\hline 11.4 & 12.1 & 0 \\
\hline 15 & 12.1 & 10.45 \\
\hline 20 & 12.1 & 17.47 \\
\hline 25 & 12.1 & 23.47 \\
\hline
\end{tabular}

Table 3 Specifications for simulation cases

\begin{tabular}{|c|c|c|c|}
\hline Case & Platform Motion & Wind Speed & Rotor Speed \\
\hline 1 & Fixed & \multirow{2}{*}{$11.4 \mathrm{~m} / \mathrm{s}$} & \multirow{2}{*}{$12.1 \mathrm{RPM}$} \\
\hline 2 & Surge: Amplitude $=2 \mathrm{~m}$, Period =12 $\mathrm{s}$ & & \\
\hline
\end{tabular}

Table 4 Wind turbine aerodynamic thrust and power under fixed condition (percentage in parentheses shows difference of flexible case over rigid case for OpenFOAM and FAST v8 simulations, respectively)

\begin{tabular}{|c|c|c|}
\hline & Thrust $(\mathrm{kN})$ & Power $(\mathrm{MW})$ \\
\hline Present Rigid & 746 & 5.06 \\
\hline Present Flexible & $733(-1.7 \%)$ & $4.9(-3.1 \%)$ \\
\hline FAST v8 Rigid & 725 & 5.37 \\
\hline FAST v8 Flexible & $701(-3.3 \%)$ & $5.16(-3.9 \%)$ \\
\hline
\end{tabular}

Table 5 Wind turbine blade tip deflection under fixed condition (percentage in parentheses shows difference of data by FAST v8 over present results)

\begin{tabular}{|c|c|c|}
\hline Solver & Present & FAST v8 \\
\hline Flapwise (m) & 5.6 & $5.52(-1.4 \%)$ \\
\hline Flapwise/Radius (-) & $8.89 \%$ & $8.76 \%$ \\
\hline Edgewise (m) & -0.6 & $-0.61(+1.7 \%)$ \\
\hline Edgewise/Radius (-) & $-0.95 \%$ & $-0.97 \%$ \\
\hline
\end{tabular}




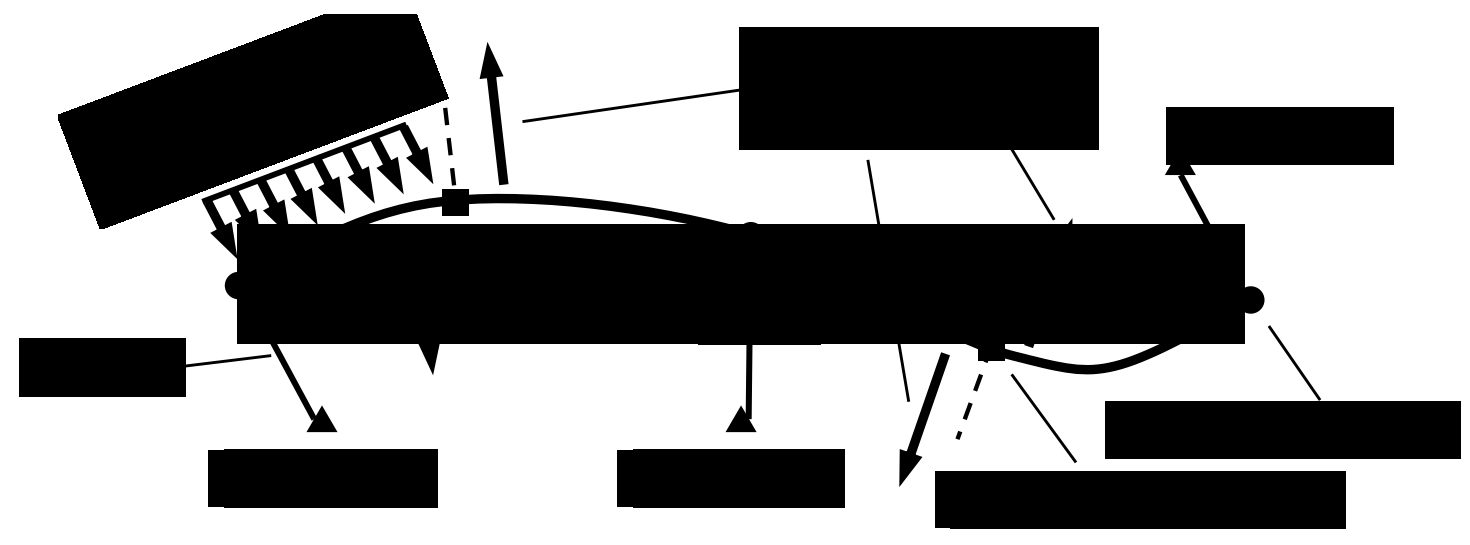

Figure 1 Illustration of a three-node beam in MBDyn

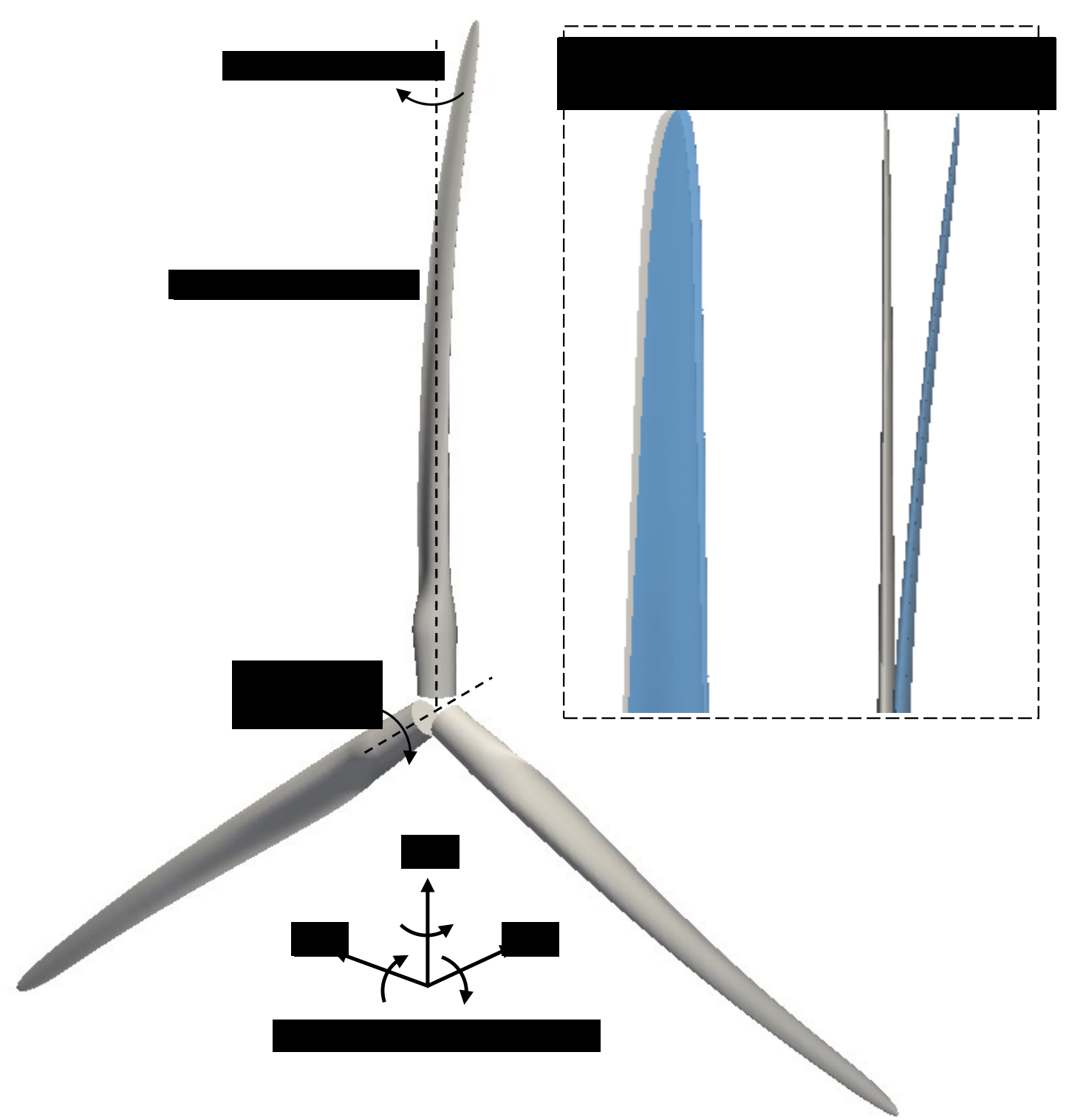

Figure 2 Illustration of complex structural responses involved in FOWT simulations with flexible blades 


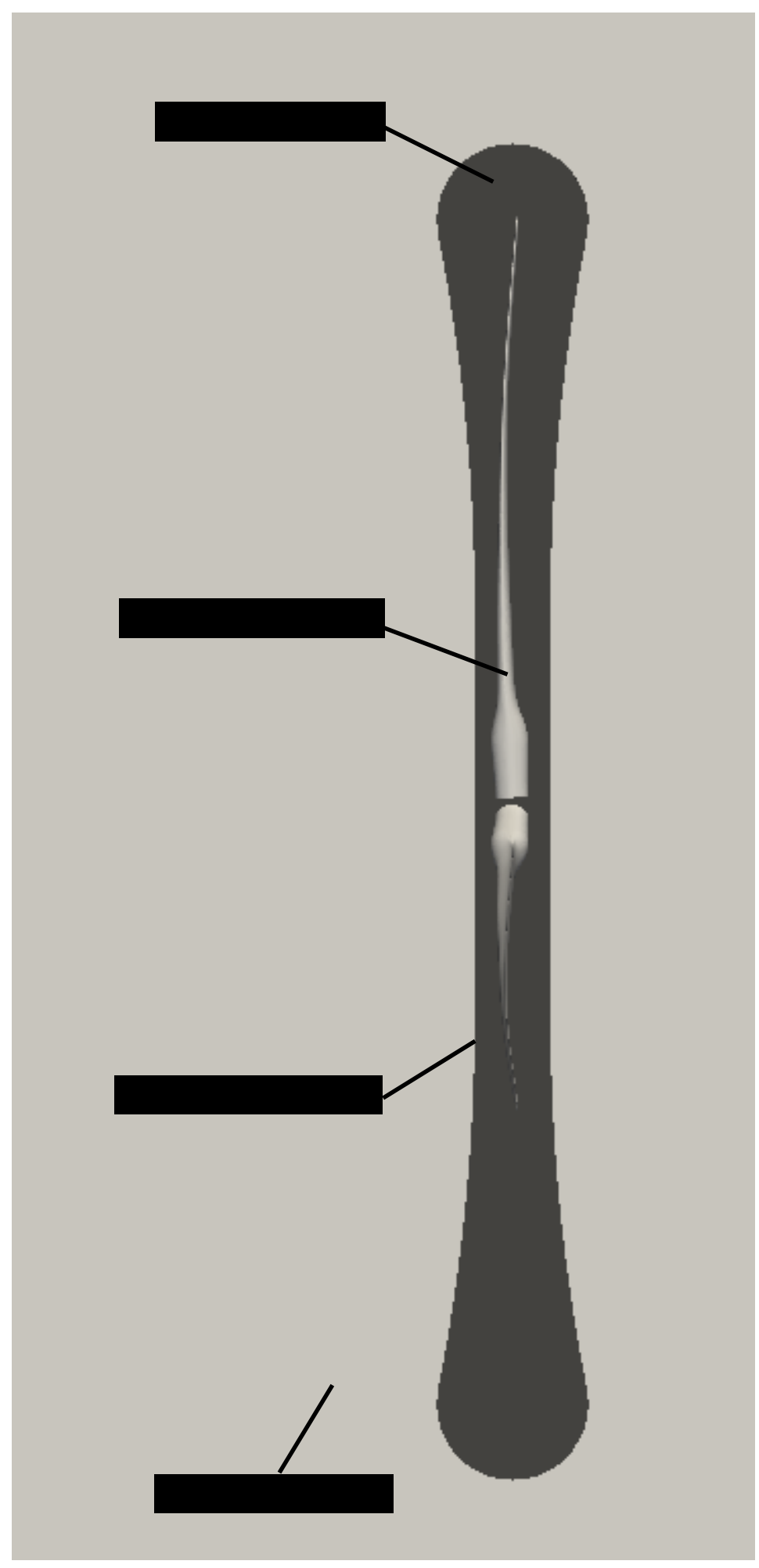

Figure 3 Cell zones for wind turbine simulations 

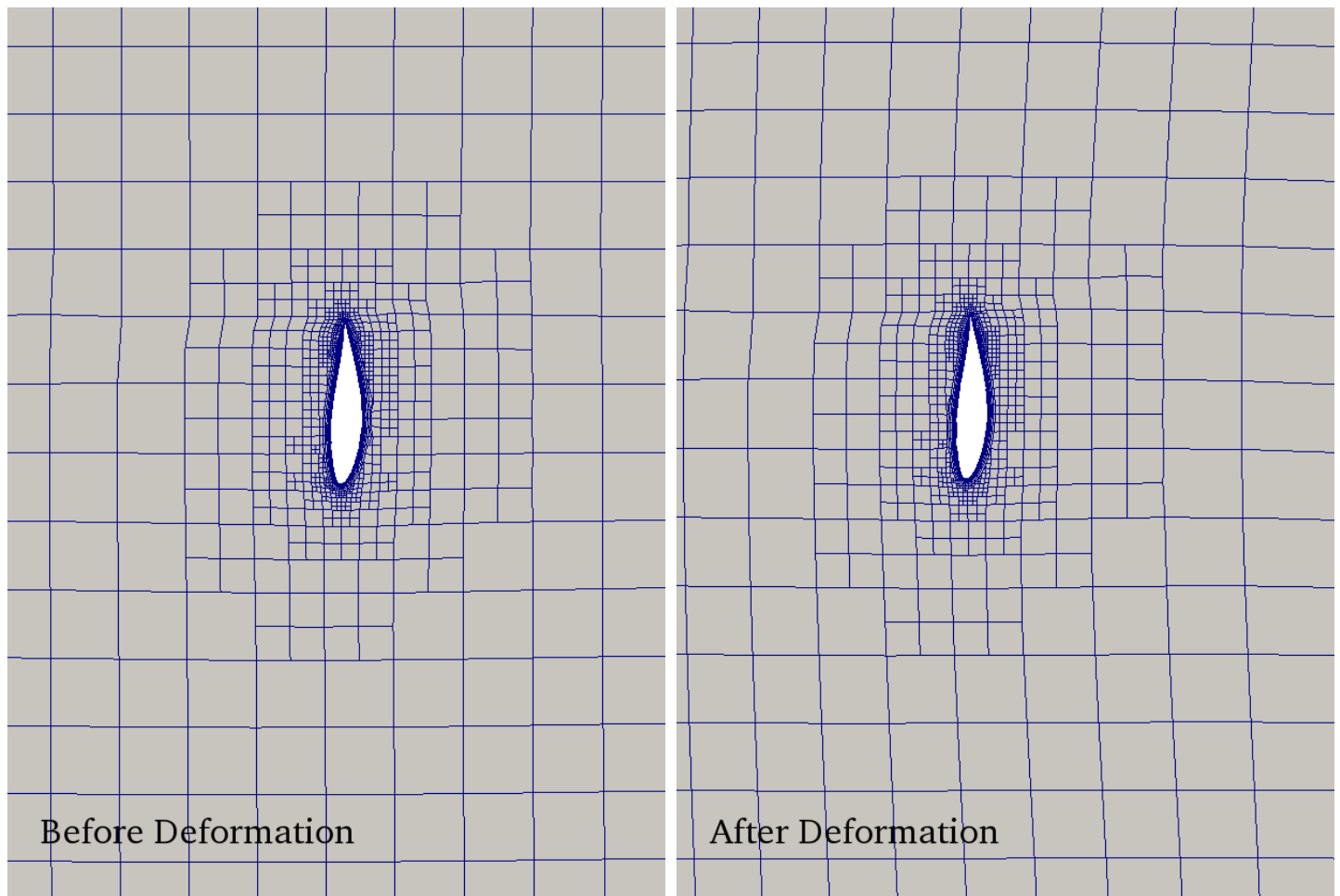

Figure 4 Comparison of mesh before and after deformation 


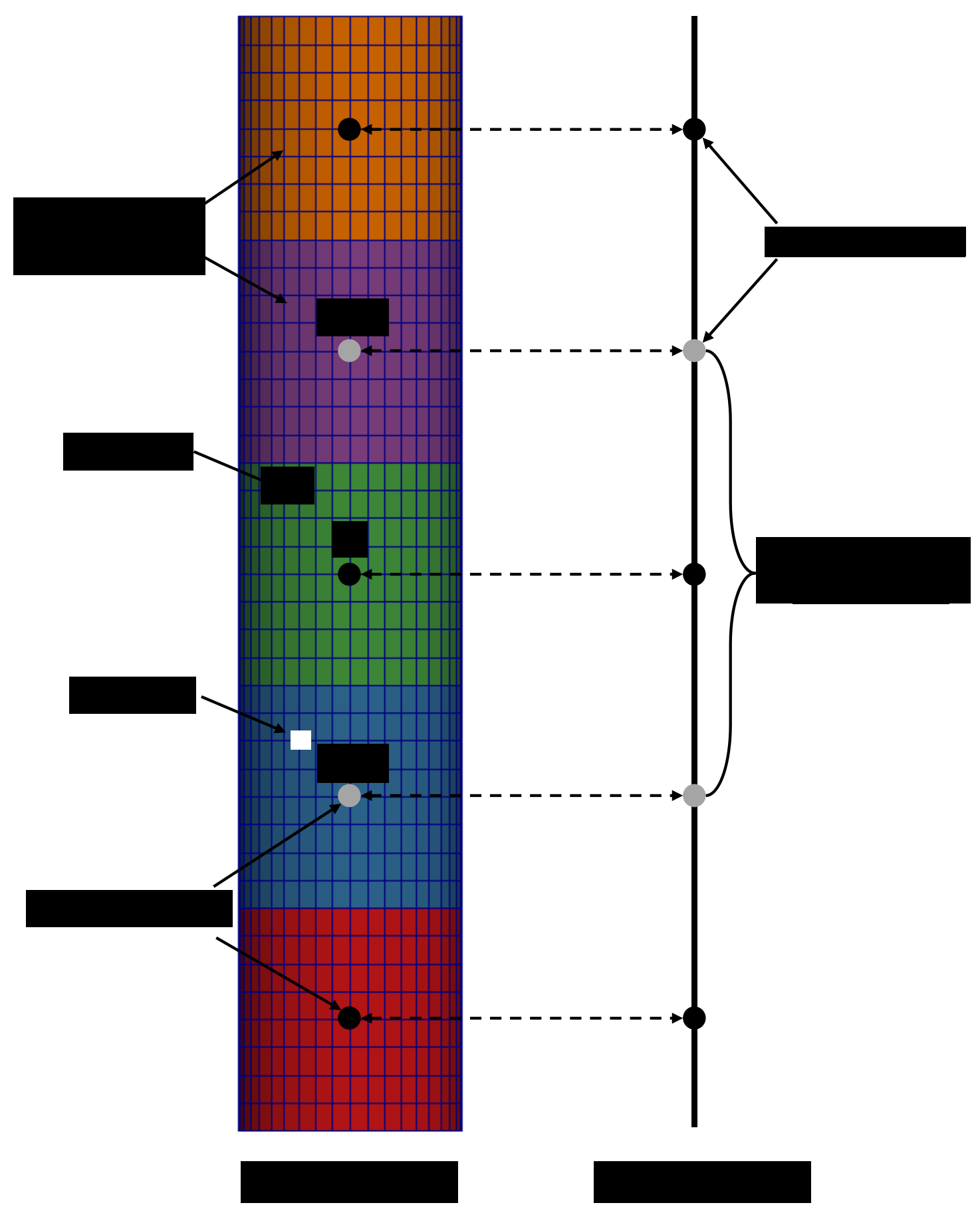

Figure 5 Diagram for mapping information between CFD and MBD models 


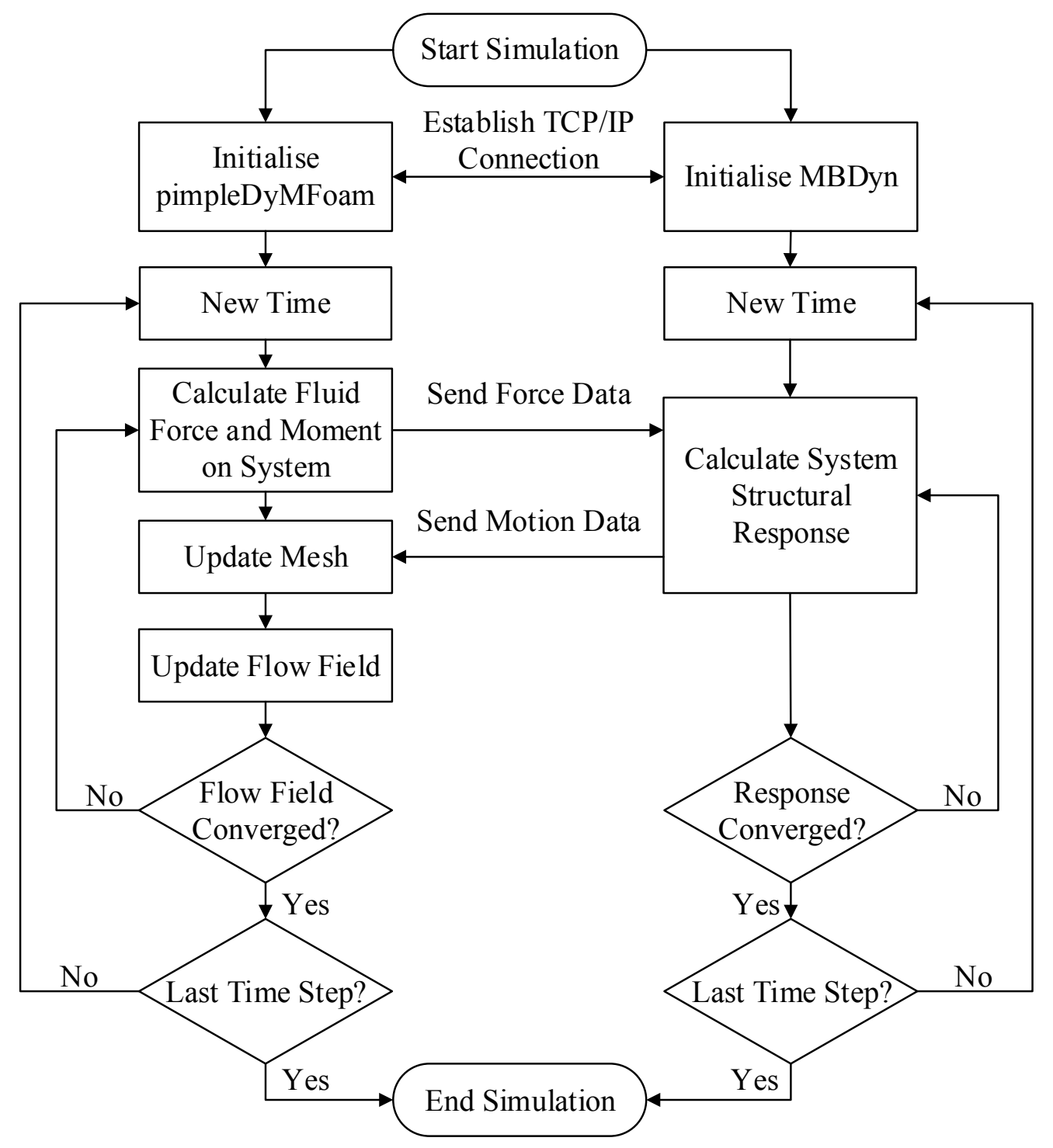

Figure 6 Flowchart for coupling pimpleDyMFoam with MBDyn 


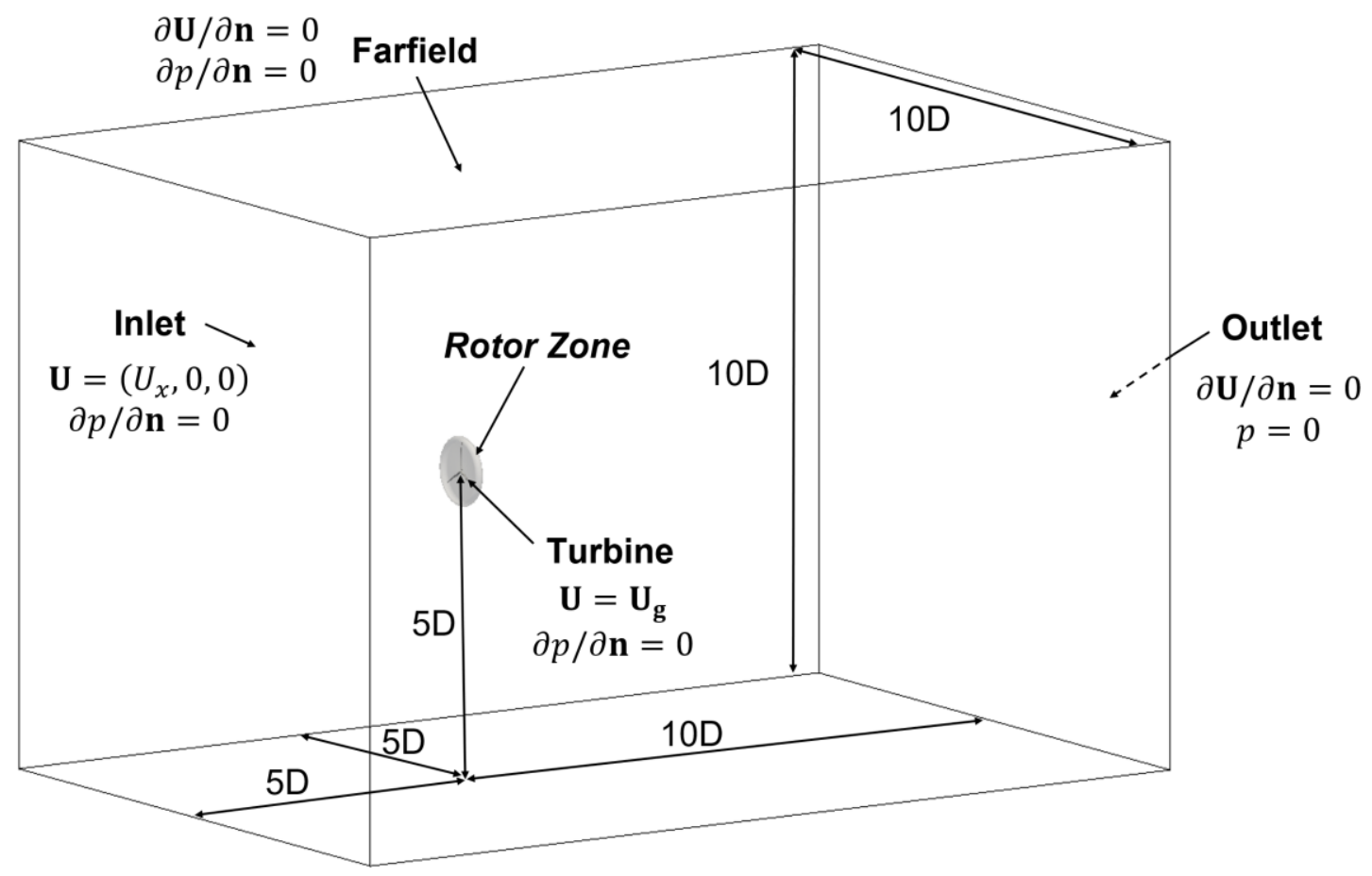

Figure 7 Dimensions and boundary conditions for wind turbine simulations: D - rotor diameter

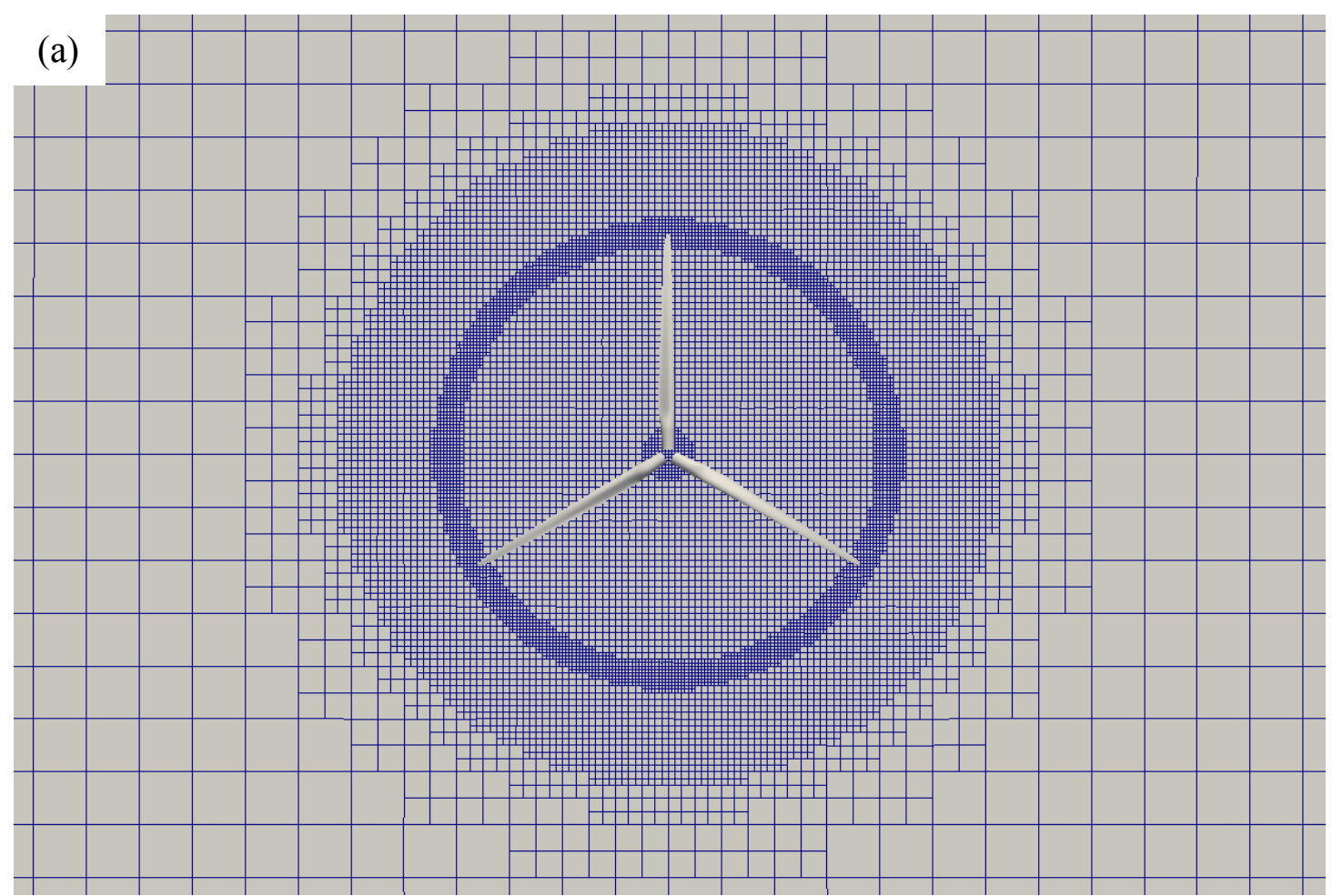


(b)

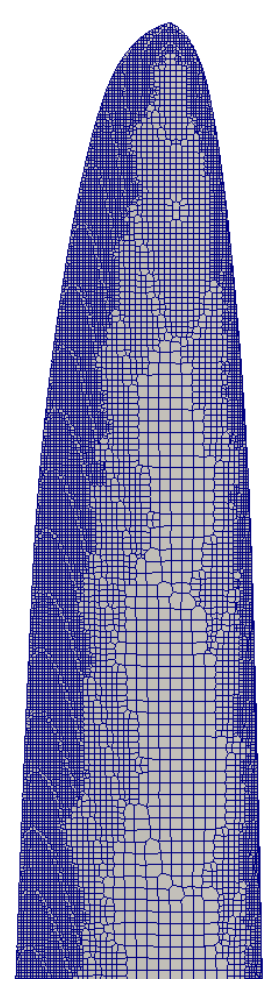

(c)

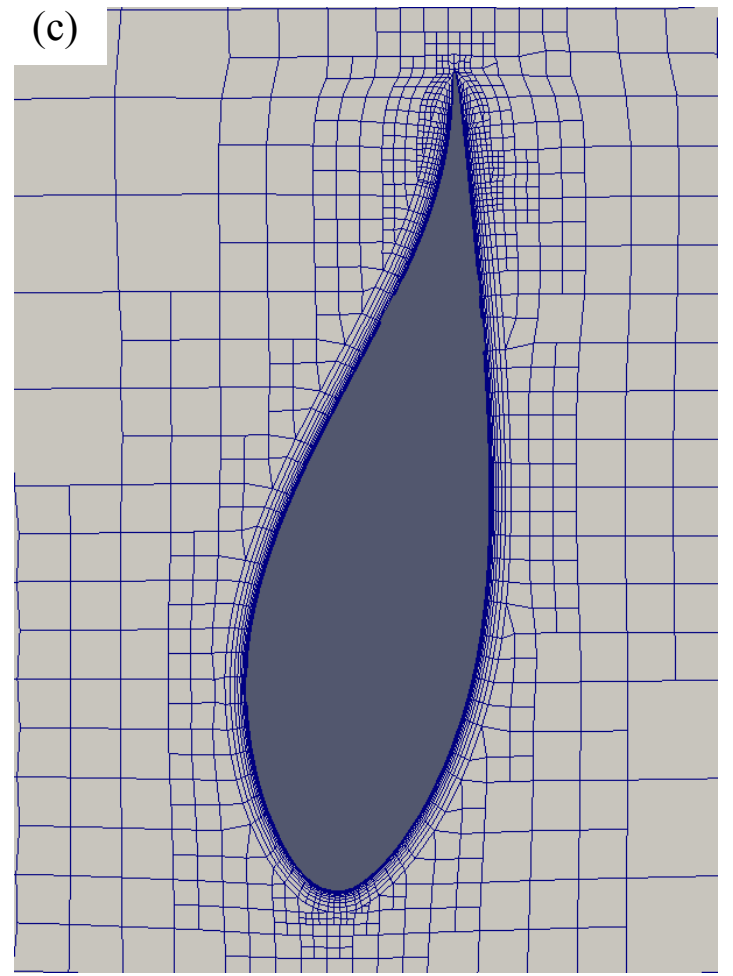

Figure 8 Computational mesh (Grid \#3) of wind turbine: (a) mesh refinement at blade root and tip, (b) blade surface mesh and (c) boundary layer mesh

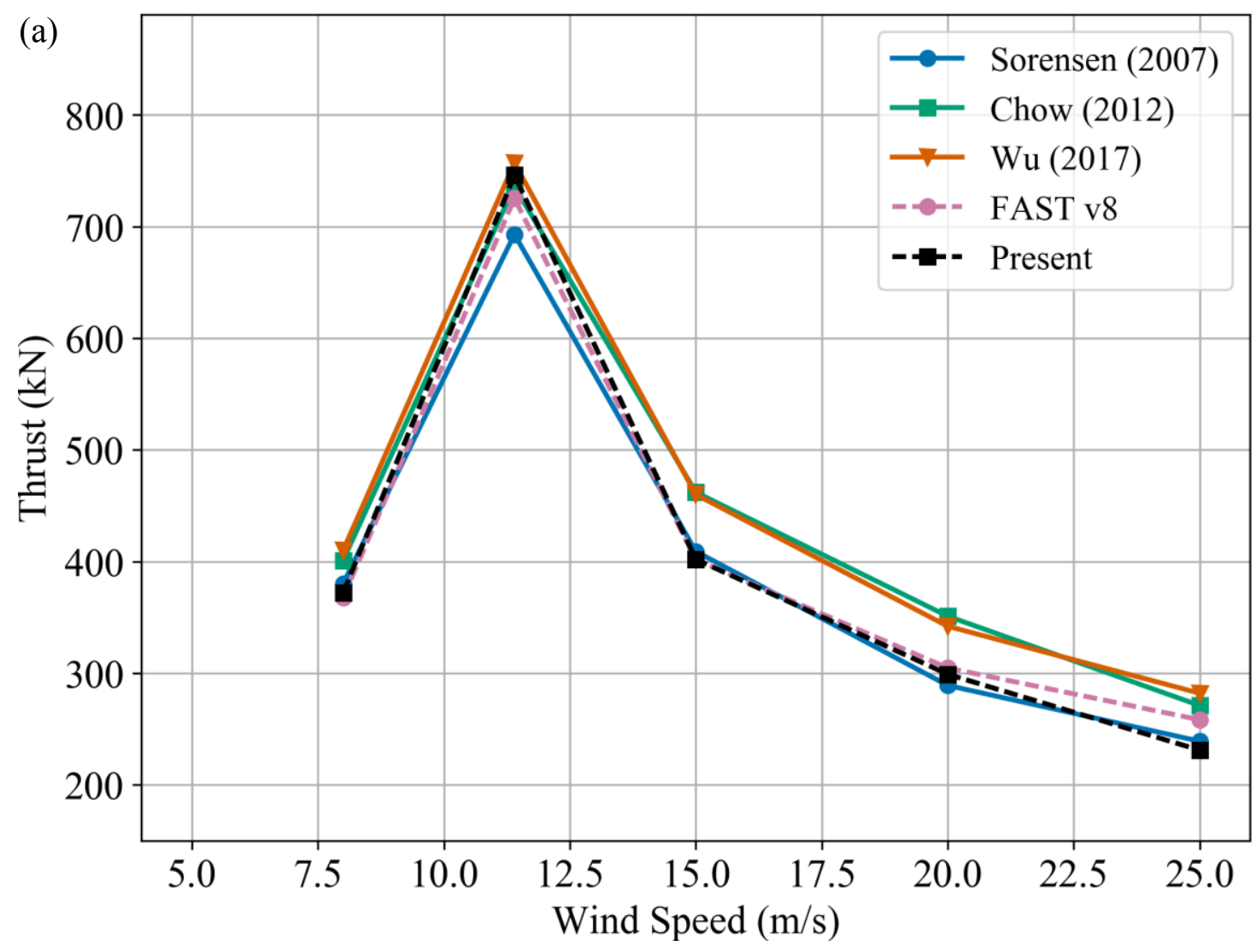




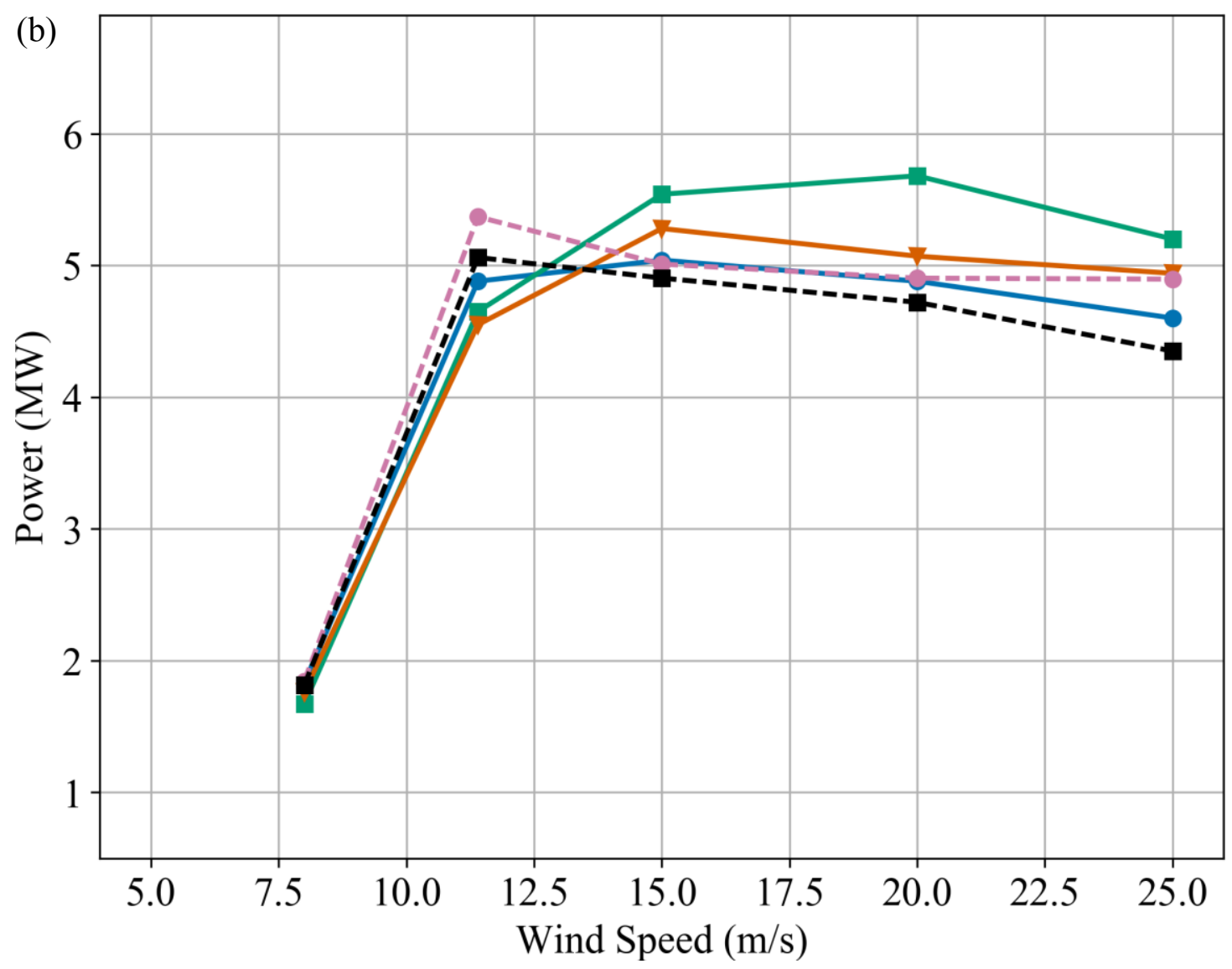

Figure 9 Wind turbine aerodynamic performance for various working conditions used in benchmark tests: (a) thrust and (b) power

\section{3-Node Beam Element}

\section{Beam Node}

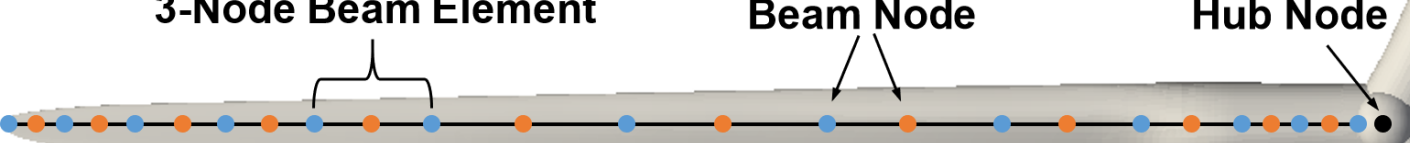

Figure 10 Structural model of wind turbine blade 

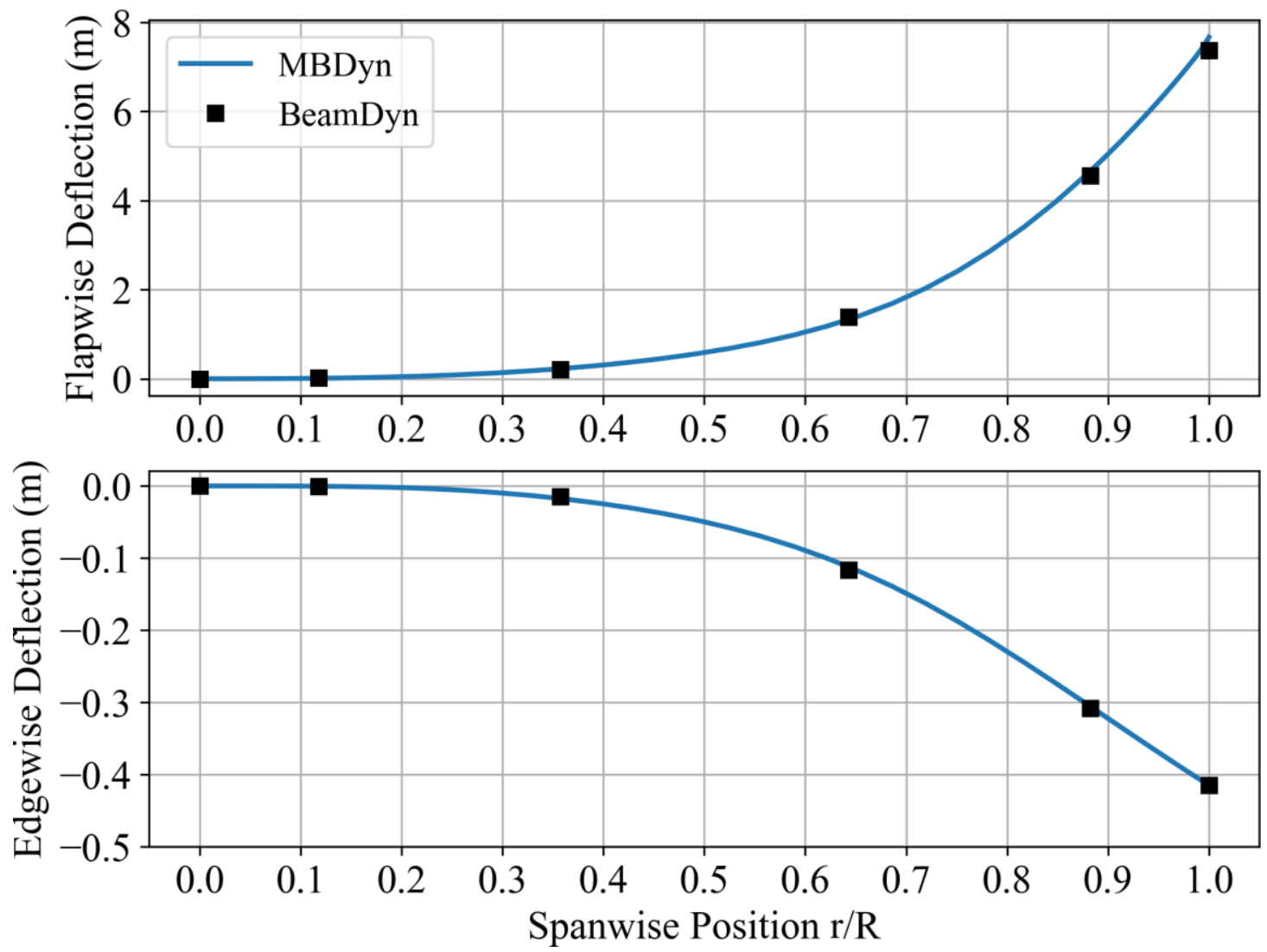

Figure 11 Blade deflection under single concentrated loading of $100 \mathrm{kN}$ at tip in flapwise direction

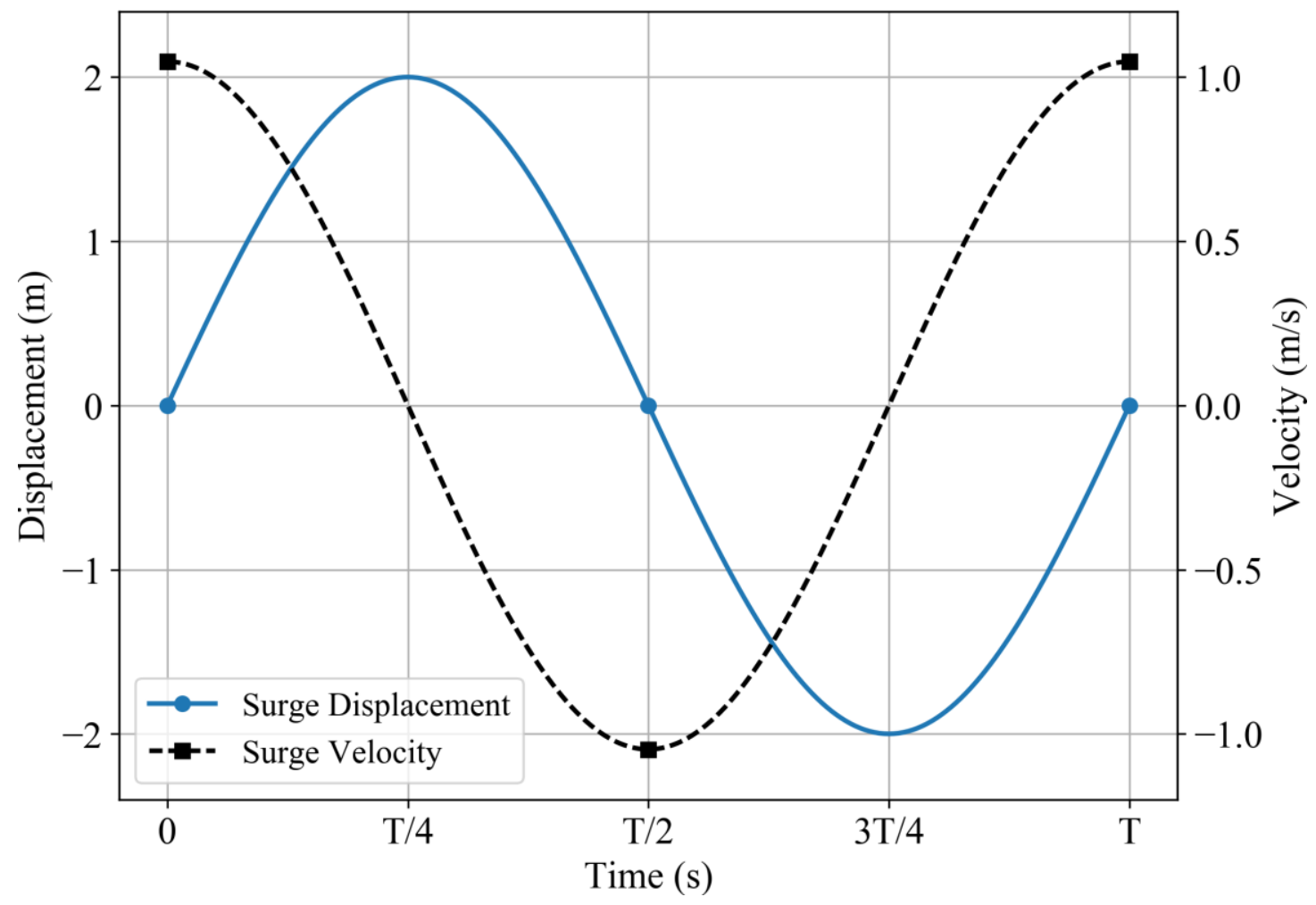

Figure 12 Prescribed platform surge displacement and velocity over one motion cycle 

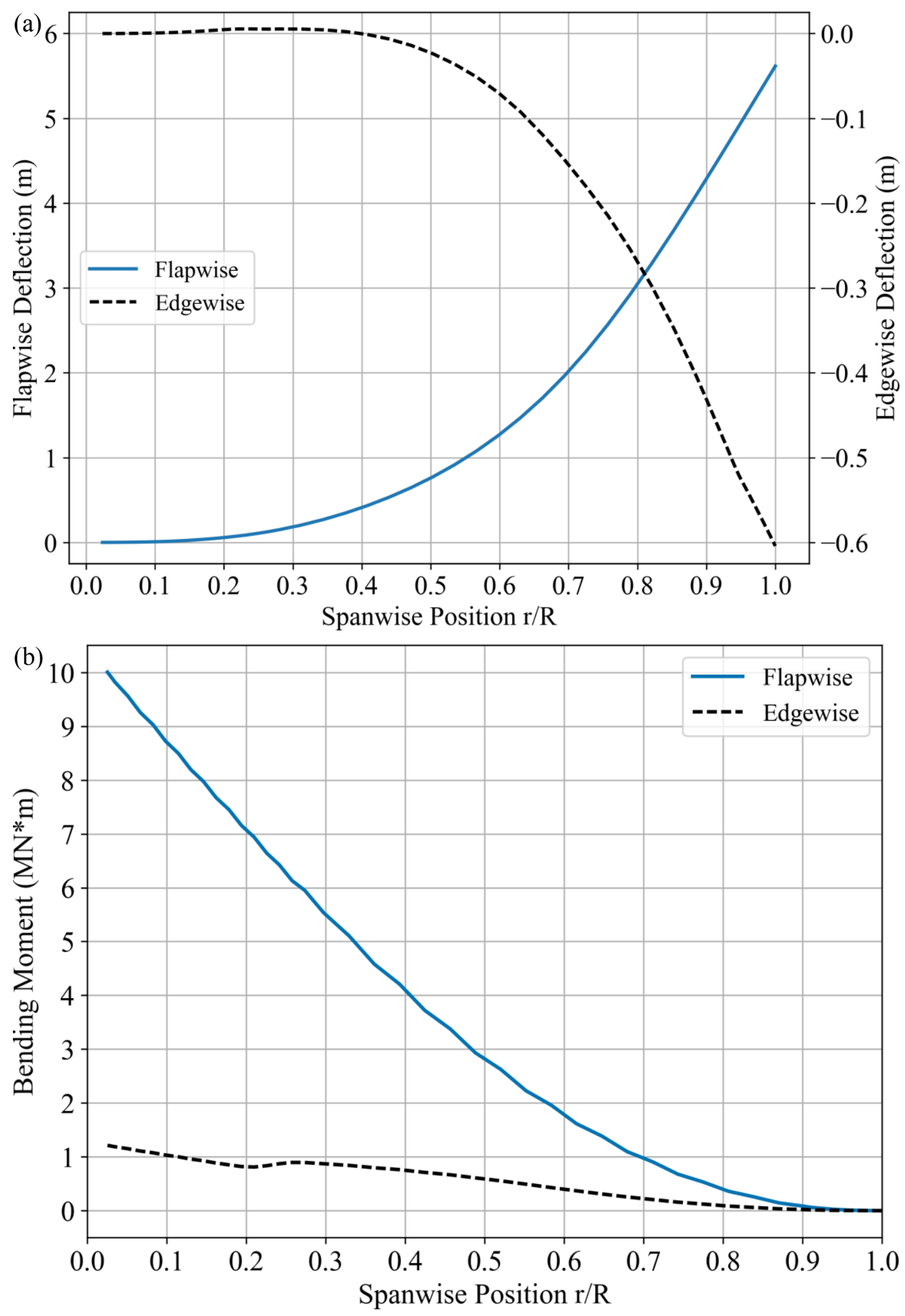

Figure 13 Spanwise distribution of (a) blade deflection and (b) blade bending moment in flapwise and edgewise directions under fixed condition 

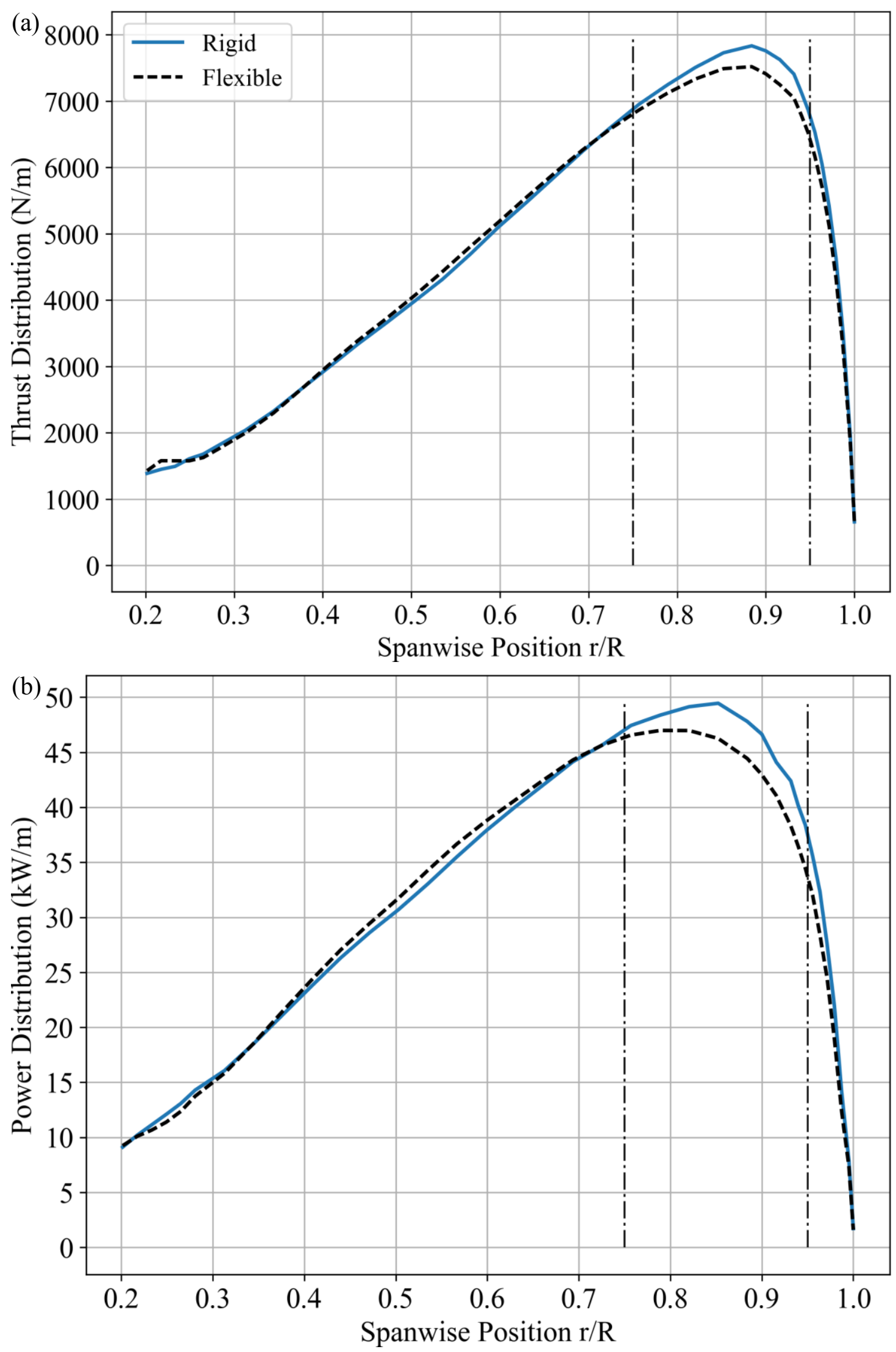

Figure 14 Spanwise distribution of (a) thrust and (b) power generated by one blade under fixed condition 

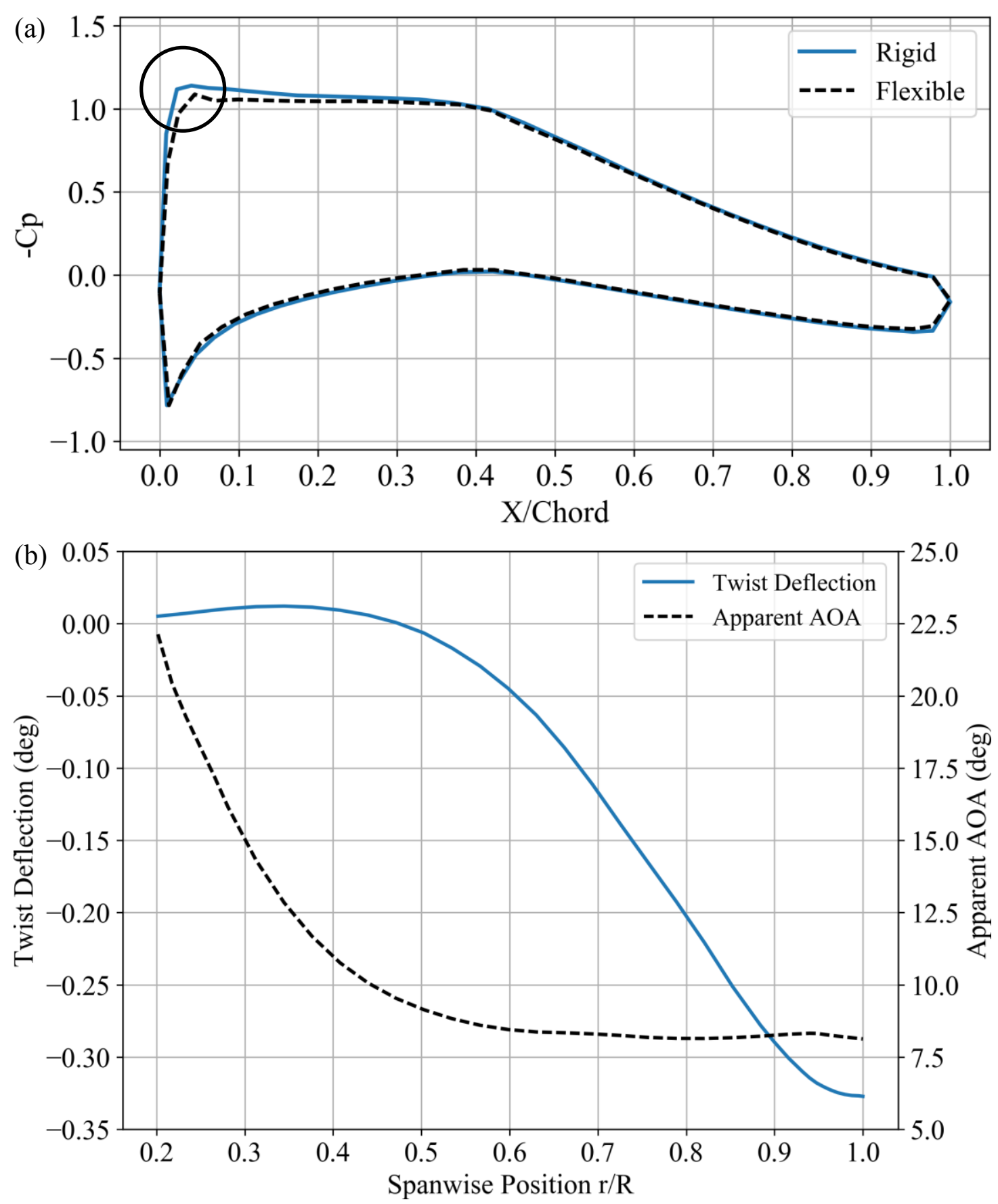

(c)

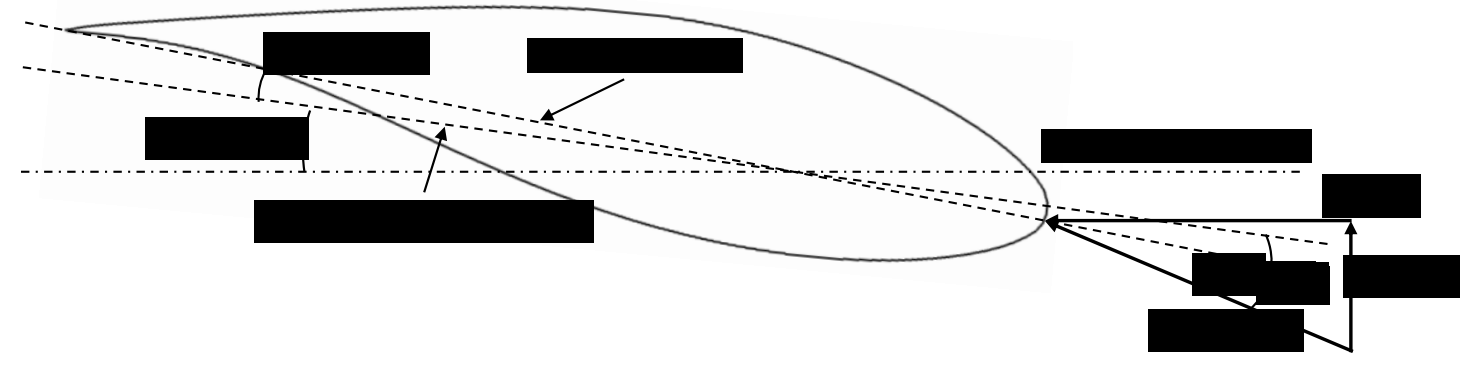

Figure 15 (a) Pressure coefficient at spanwise section of $r / R=0.9$, (b) spanwise distribution of blade twist deflection and (c) definition of apparent AOA under fixed condition: Twist ${ }_{\text {Def }}$ - twist deflection; Twist $_{\text {Aero }}$ - aerodynamic twist; $\mathrm{U}_{\text {RelWind }}$ - relative wind speed; $\alpha$ - apparent AOA; $\alpha_{0}$ - AOA before twist deflection 

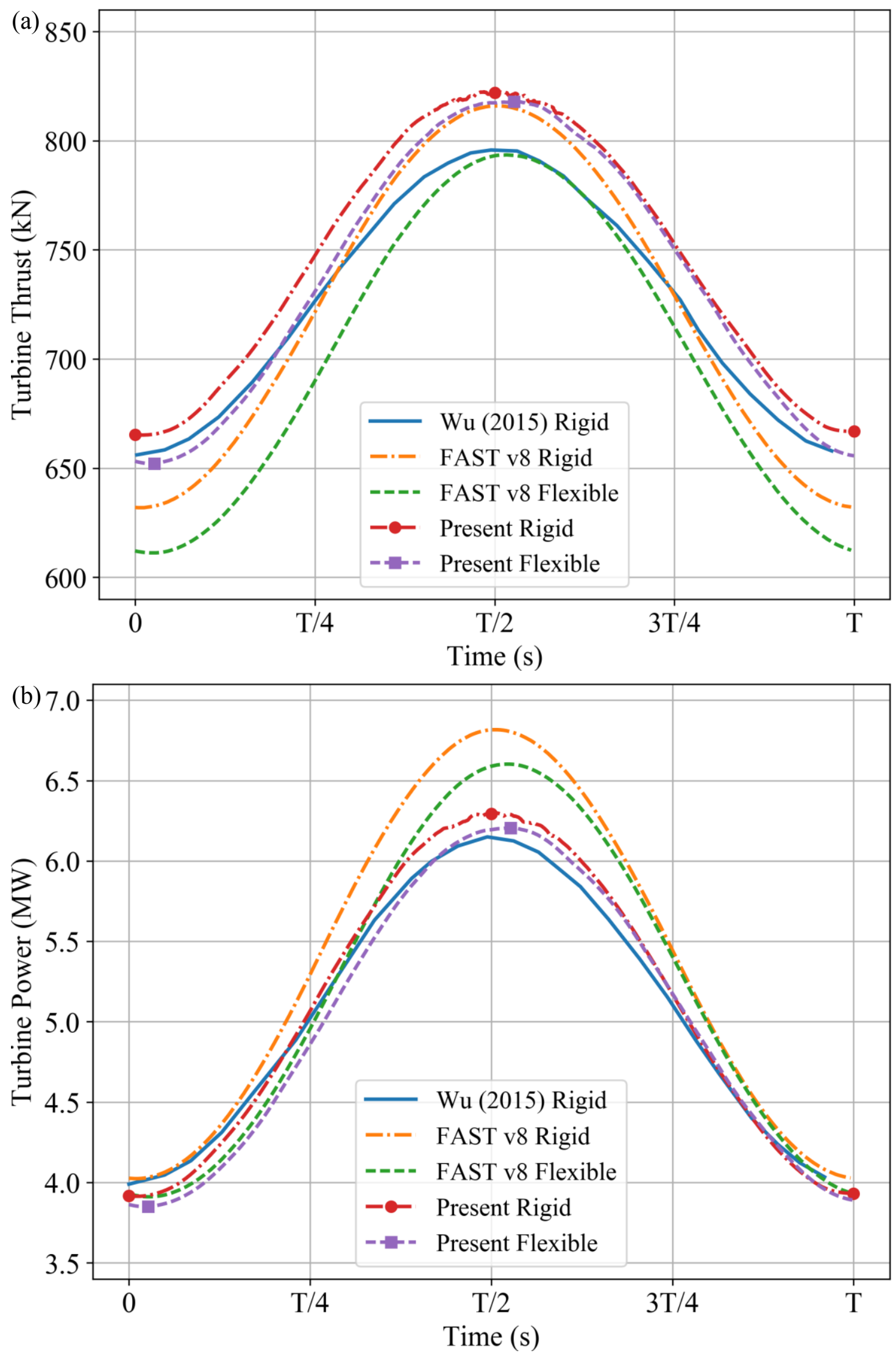

Figure 16 Time history of wind turbine (a) thrust and (b) power under prescribed platform surge motion 


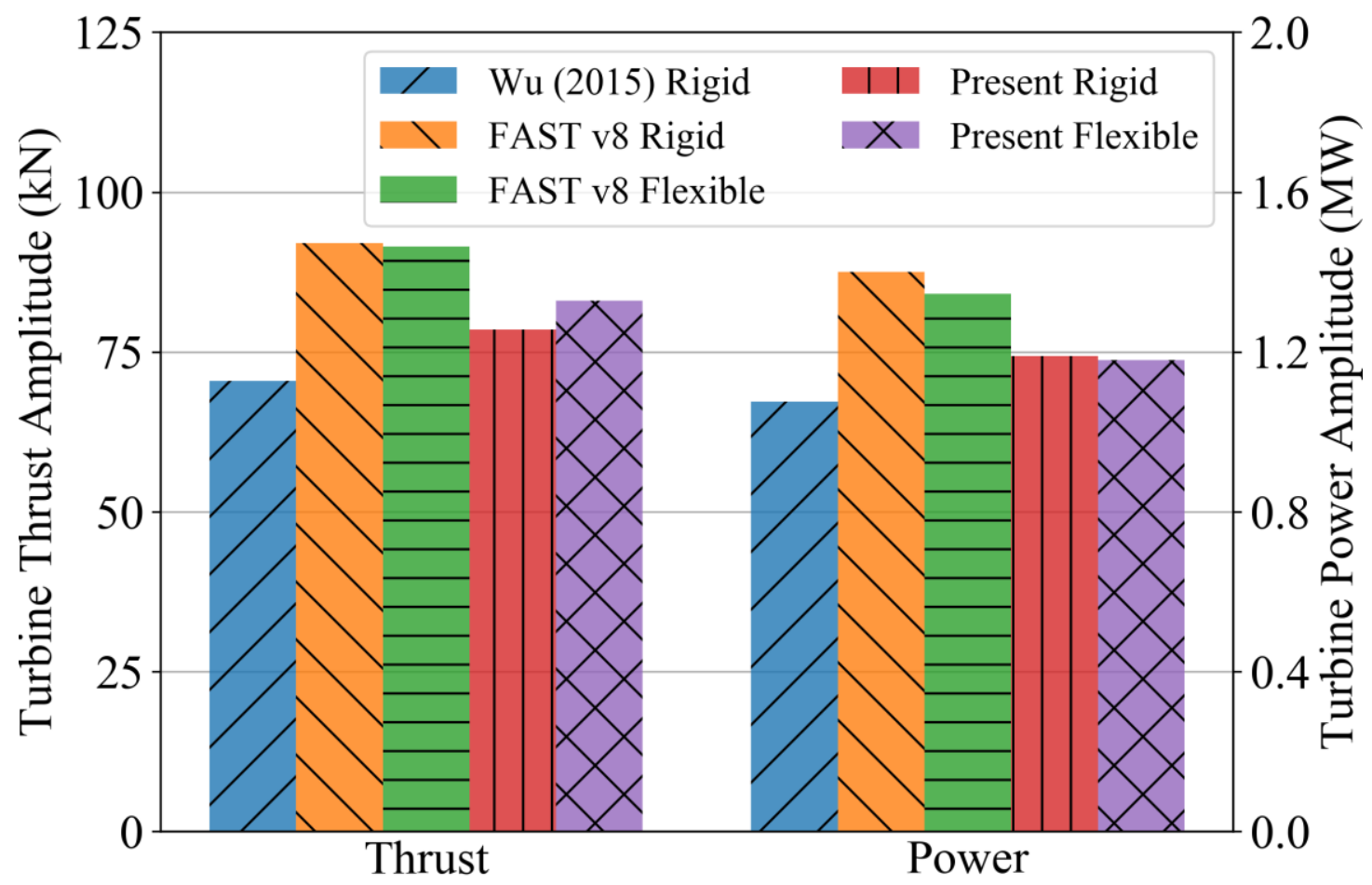

Figure 17 Variation amplitude of wind turbine thrust and power under prescribed platform surge motion

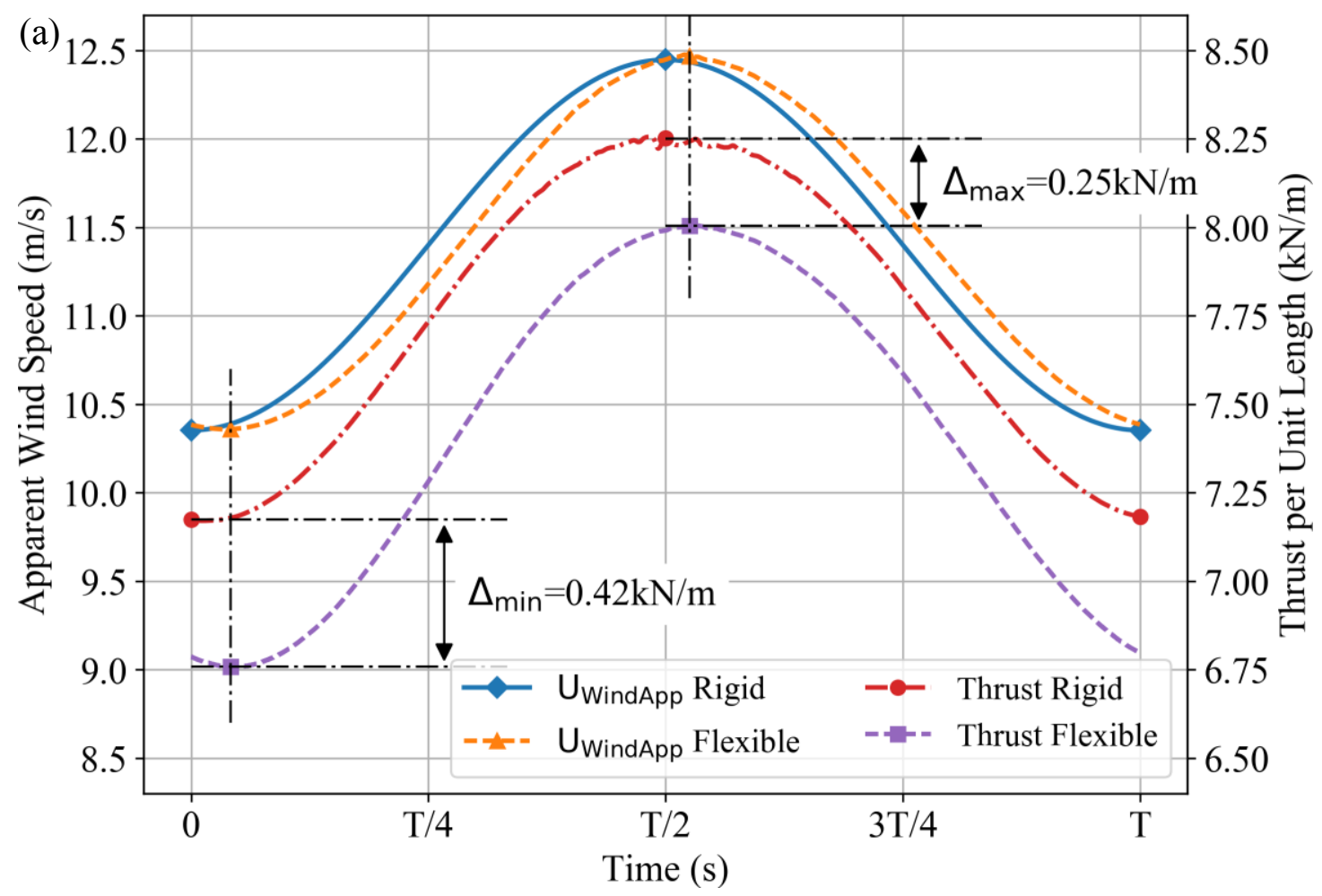




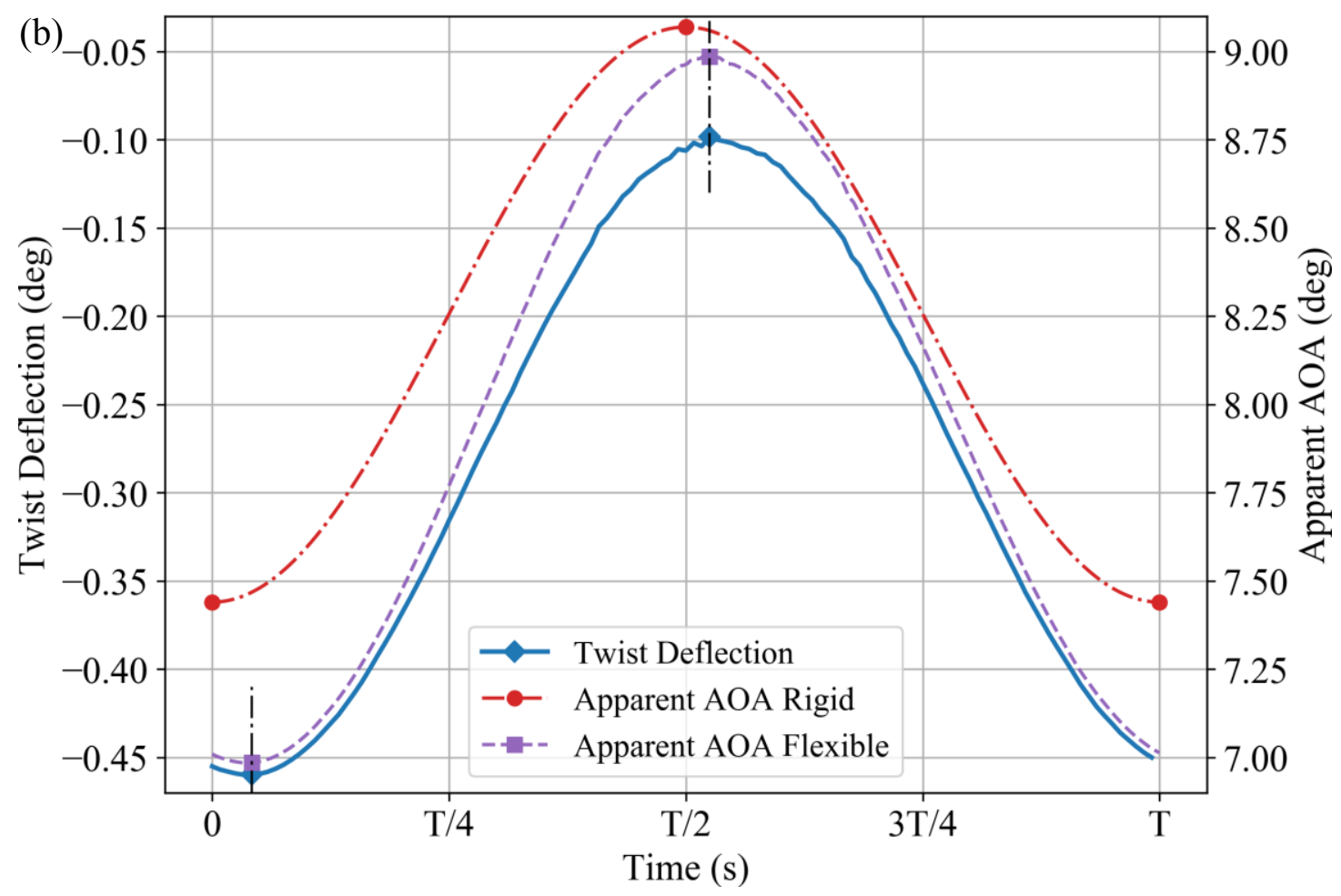

Figure 18 Time history of (a) thrust per unit length and (b) twist deflection at spanwise section of $r / R=0.9$ under prescribed platform surge motion

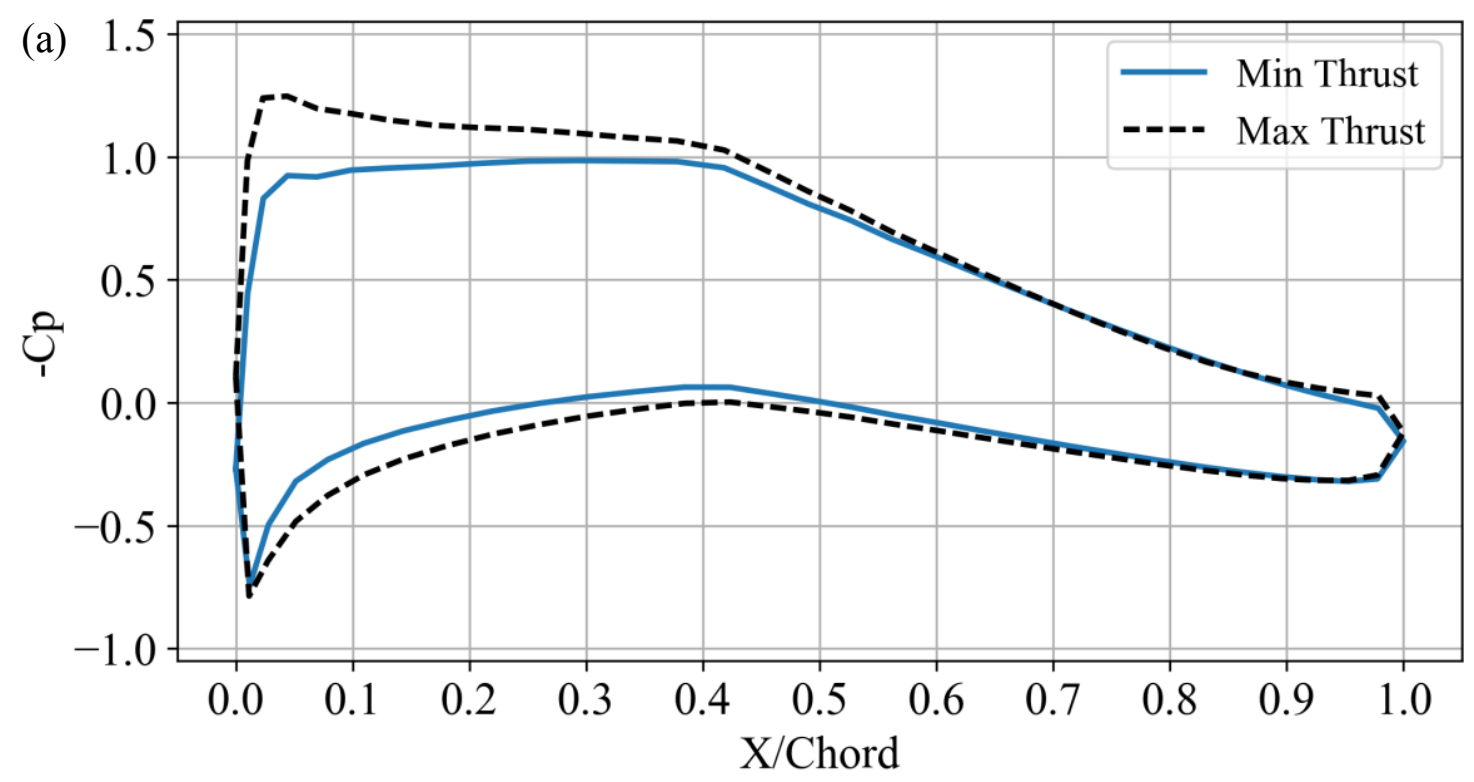




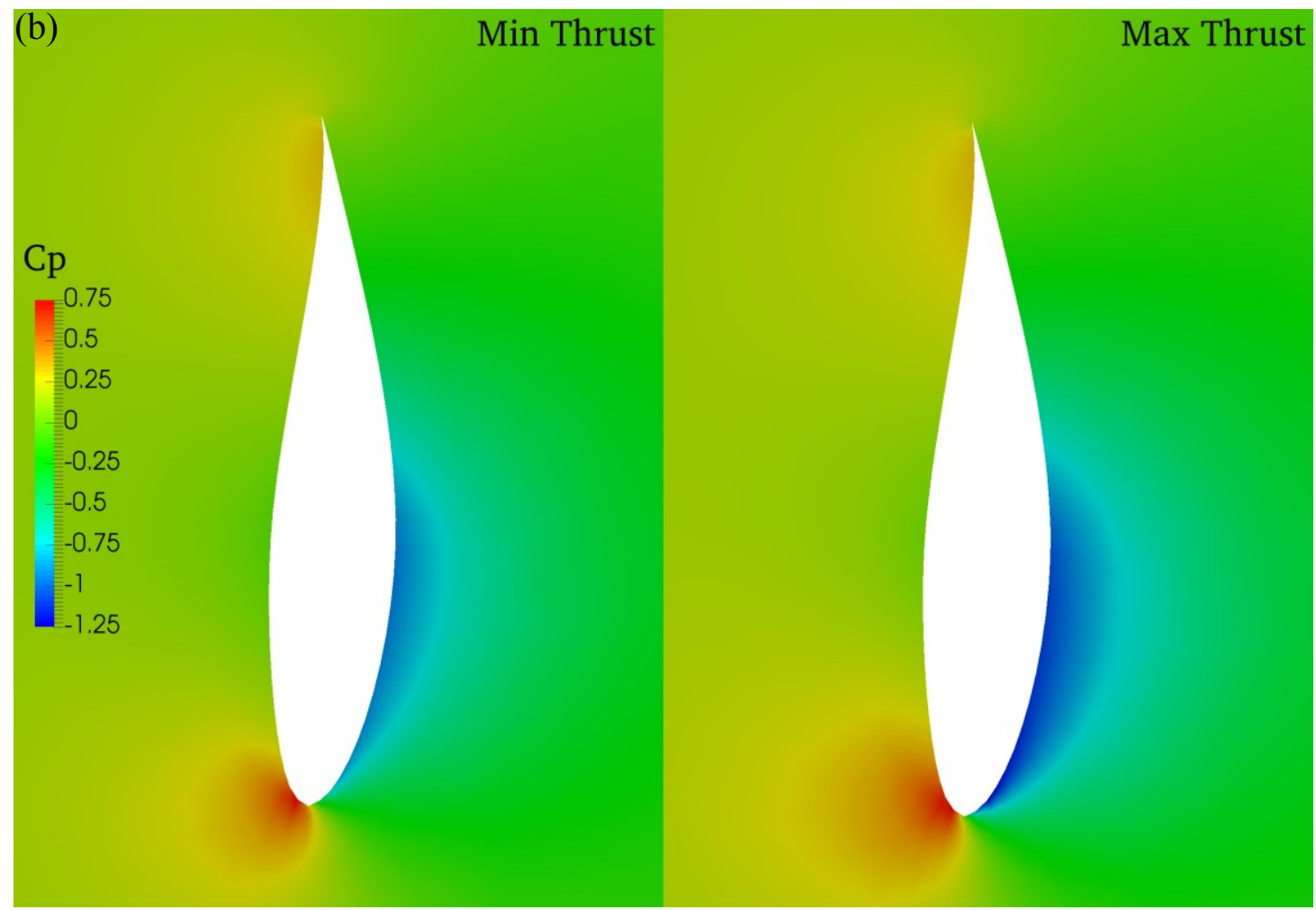

Figure 19 (a) Pressure coefficient distribution and (b) fluid field coloured by pressure coefficient for flexible case at spanwise section of $\mathrm{r} / \mathrm{R}=0.9$ under prescribed platform surge motion: Min Thrust - At time of minimum thrust per unit length; Max Thrust At time of maximum thrust per unit length

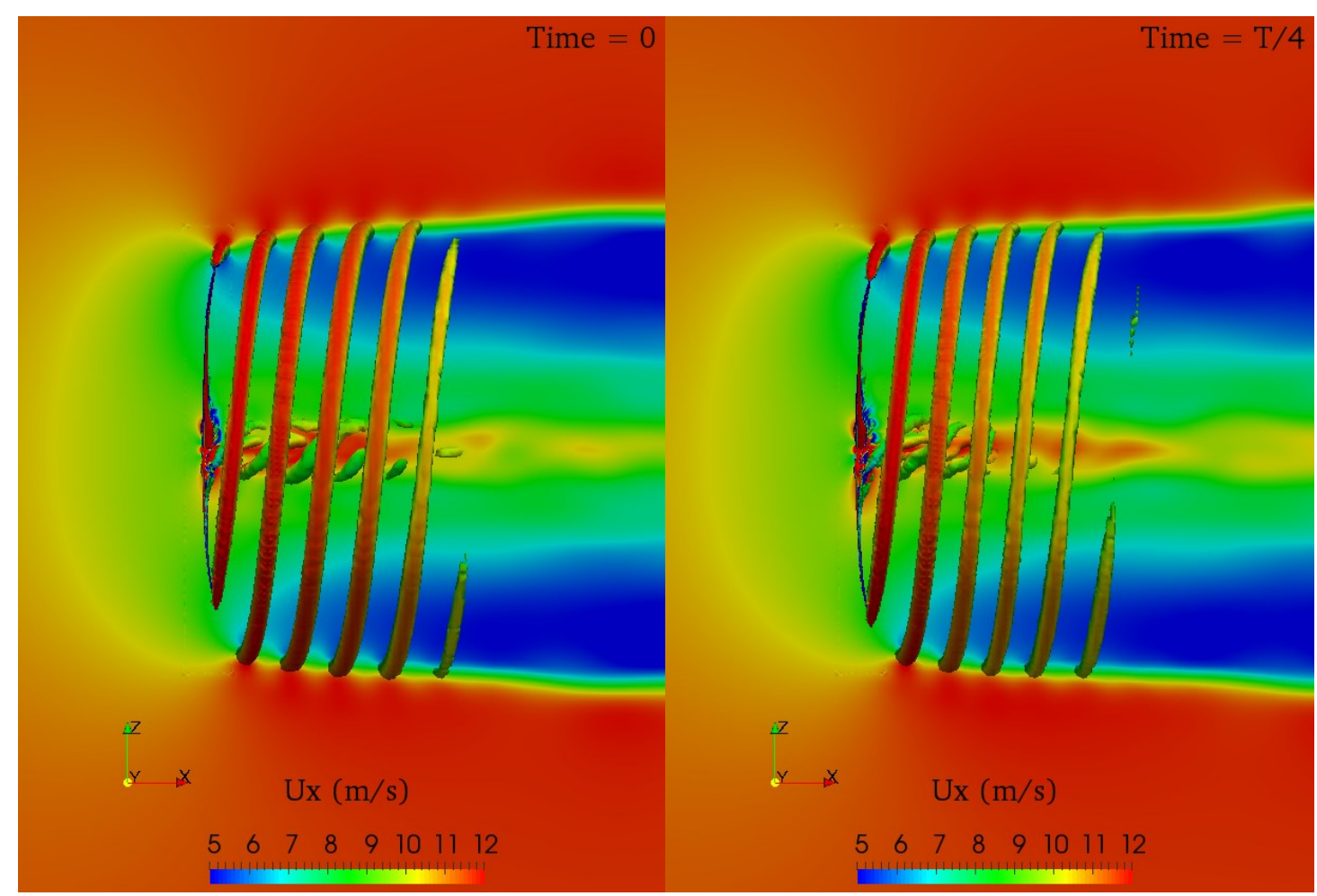




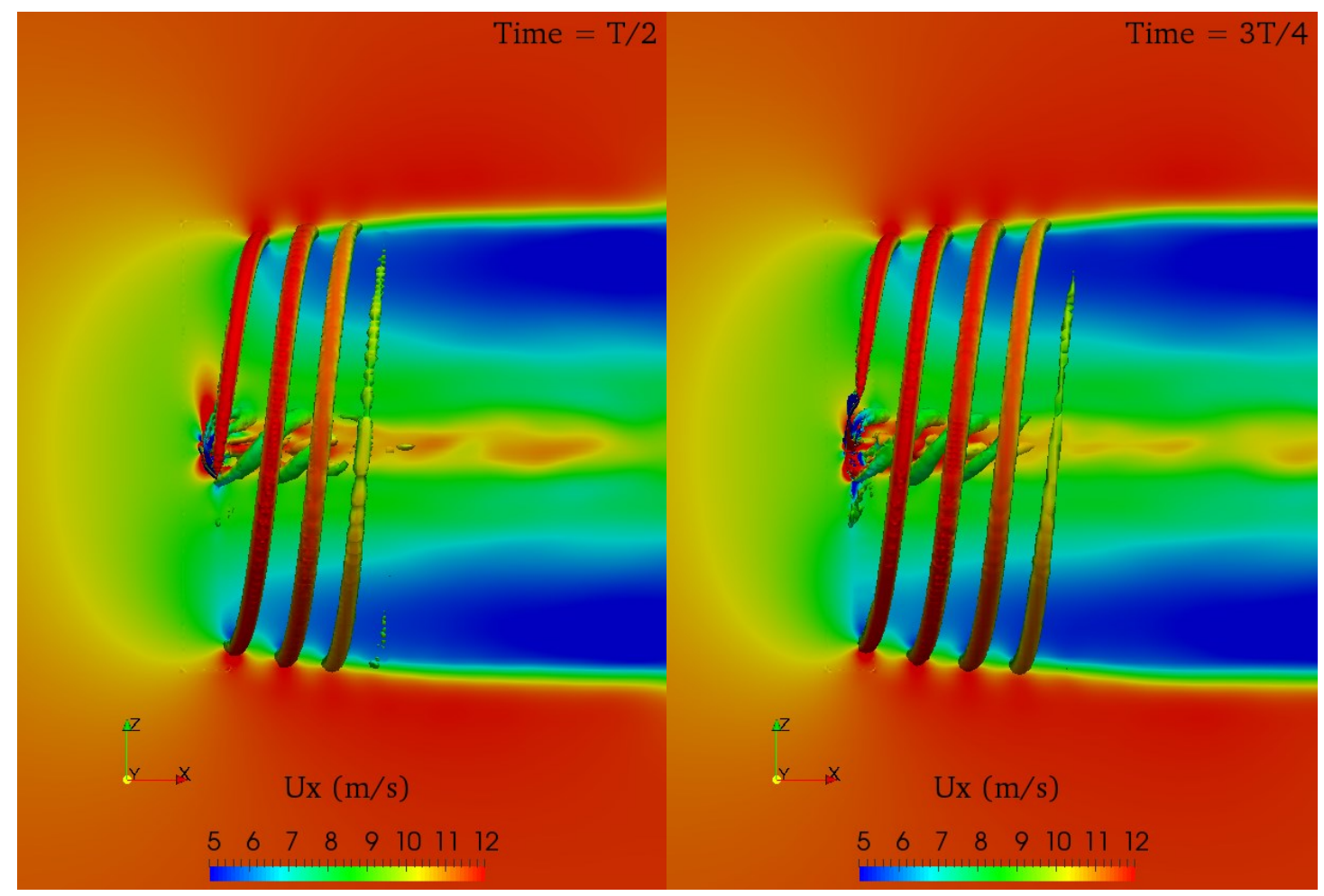

Figure 20 Vortex contour $(\mathrm{Q}=0.1)$ and flow field at mid-plane coloured by axial velocity $U x$ under prescribed platform surge motion
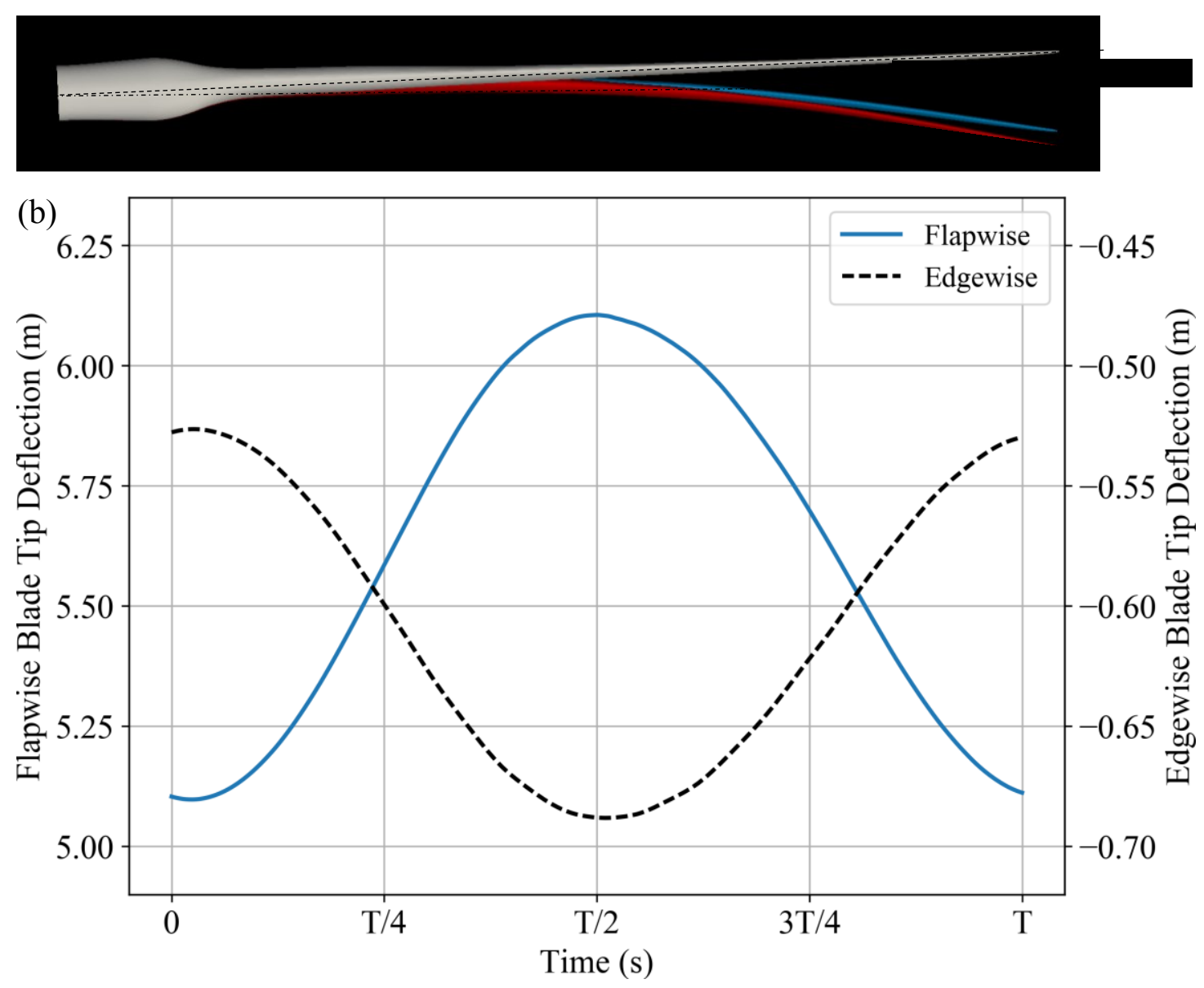


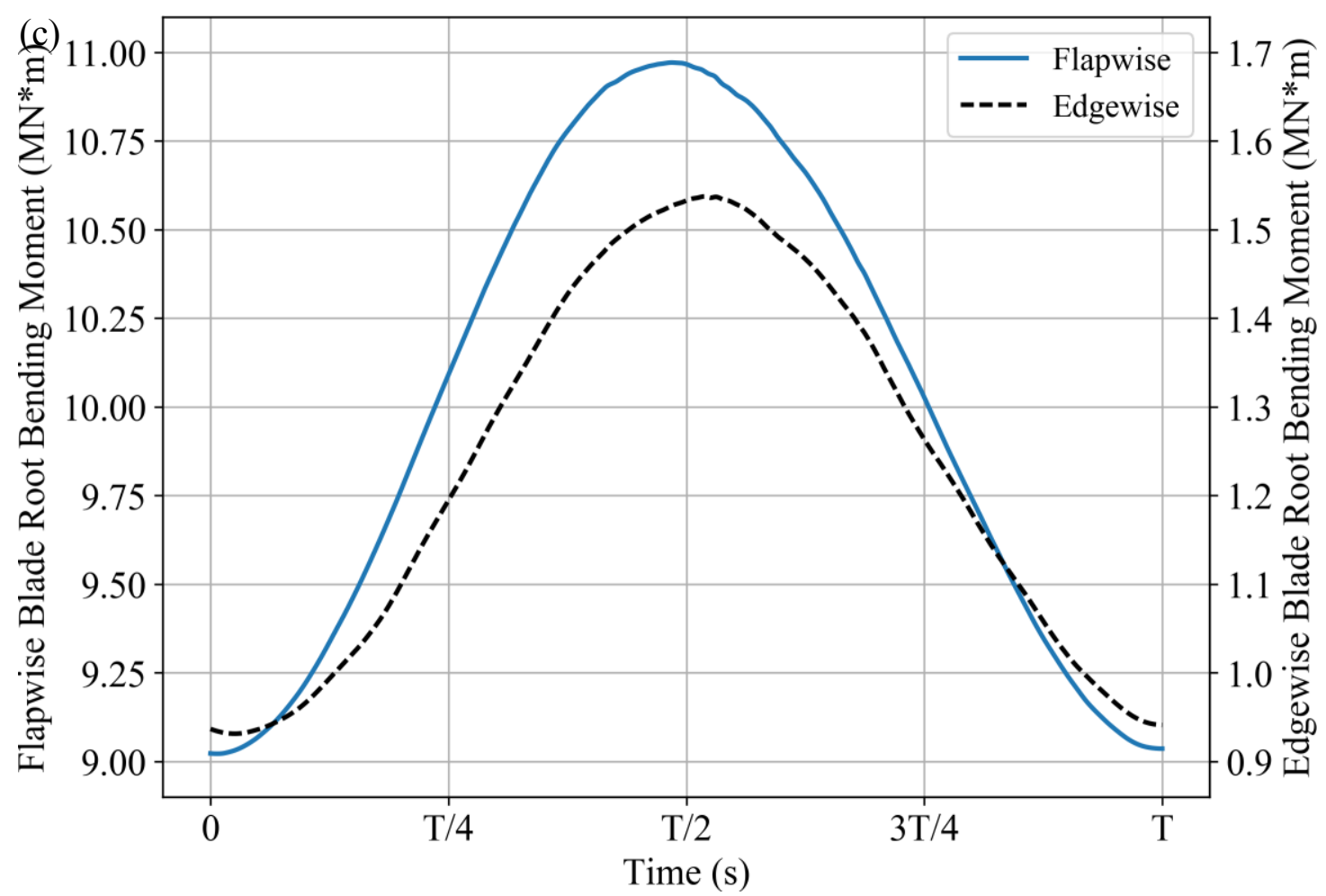

Figure 21 (a) Illustration of blade deflection, (b) time history of flapwise and edgewise blade tip deflection and (c) blade root bending moment under prescribed platform surge motion 